\title{
Integrated Semigroups on Fréchet Spaces I: Bochner Integral, Laplace Integral and Real Representation Theorems
}

Tiziano Granucci

Correspondence: Istituto Superiore Leonardo da Vinci, Via del Terzolle 91, 50127, Firenze, Italy. E-mail: tizianogranucci@isisidavinci.eu

Received: December 27, 2018 Accepted: January 22, 2019 Online Published: January 27, 2019

doi:10.5539/jmr.v11n1p118～URL: https://doi.org/10.5539/jmr.v11n1p118

Abstract

In this articles we will study the integration of the vectorial functions in Fréchet spaces. Particularly we will introduce and we will study a new functional space and we will prove some theorems of representation.

Keywords: Fréchet spaces, semigroups, Laplace transforms

\section{Preliminaries}

In this articles we will study the integration of the vectorial functions in Fréchet spaces. Particularly we will introduce and we will study a new functional space and we will prove some theorems of representation.

\subsection{Definitions}

Definition 1. A real-valued function $p(x)$ defined on a complex linear space $S$ is called a semi-norm, if

$$
p(x+y) \leq p(x)+p(y) \quad \forall x, y \in S
$$

and

$$
p(\lambda x)=|\lambda| p(x) \quad \forall \lambda \in \mathbb{C}, \forall x \in S .
$$

Definition 2. A complex linear topological spaces $S$ is called a locally convex, linear topological space, or, in short, a locally convex space, if any if its open sets contains a convex, balanced and absorbing open set.

Definition 3. A complex linear space $\mathfrak{F}$ is called a Quasi-normed linear space if, for every $x \in \mathfrak{F}$, there is associated a real number $|x|_{\widetilde{\wp}}$, the quasi-norm of the vector $x$, which satisfies

$$
\begin{gathered}
|x|_{\mathfrak{F}} \geq 0 \text { and }|x|_{\mathfrak{F}}=0 \Leftrightarrow x=0 ; \\
|x+y|_{\mathfrak{F}} \leq|x|_{\mathfrak{F}}+|y|_{\mathfrak{F}} \quad \forall x, y \in \mathfrak{F} ; \\
|x|_{\mathfrak{F}}=|-x|_{\mathfrak{F}} \quad \forall x \in \mathfrak{F} ; \\
\lim _{\alpha_{n} \rightarrow 0}\left|\alpha_{n} x\right|_{\mathfrak{F}}=0 \quad \forall x \in \mathfrak{F} ; \\
\lim _{|x|_{\mathfrak{F}} \rightarrow 0}|\alpha x|_{\mathfrak{F}}=0 \quad \forall \alpha \in \mathbb{C} .
\end{gathered}
$$

The topology of a quasi-normed linear space $\mathfrak{F}$ is thus defined by the distance

$$
d(x, y)=|x-y|_{\mathfrak{\wp}} .
$$

We say that the sequence $\left\{x_{n}\right\}_{n} \subset \mathfrak{F}$ converges strongly to $x \in \mathfrak{F}, x_{n} \rightarrow x$ for $n \rightarrow+\infty$ in $\mathfrak{F}$, or

$$
\mathfrak{r}-\lim _{n \rightarrow \infty} x_{n}=x,
$$

if

$$
\lim _{n \rightarrow \infty}\left|x_{n}-x\right|_{\widetilde{F}}=\lim _{n \rightarrow \infty} d\left(x_{n}, x\right)=0 .
$$




\subsection{Fréchet Space}

Definition 4. A quasi-normed linear space $\mathfrak{F}$ is called a Fréchet space if it is complete, i.e., if every Cauchy sequence of $\mathfrak{F}$ converges strongly to a point of $\mathfrak{F}$.

Remark 1. Let the topology of a locally convex space $\mathfrak{F}$ be defined by a countable number of semi-norms $p_{n}(x)$, with $n=1,2, \ldots$. Then $\mathfrak{F}$ is a quasi-normed linear space by the quasi-norm

$$
|x|_{\widetilde{r}}=\sum_{n \geq 1} \frac{p_{n}(x)}{2^{n}\left(1+p_{n}(x)\right)} .
$$

For, the convergence $\lim _{h \rightarrow \infty} p_{n}\left(x_{h}\right)=0$, with $n=1,2, \ldots$ is equivalent to $\mathfrak{F}-\lim _{h \rightarrow \infty} x_{h}=0$ with respect to the quasi-norm $|x|_{\tilde{F}}$ above.

Definition 5. (Fréchet in Bourbaki sence) A locally convex space $\mathfrak{F}$ is called a Fréchet space if it is quasi-normed and complete.

Let $X, Y$ be locally convex spaces on the same scalar field; we denote by $L(X, Y)$ the totality of continuous linear operators on $X$ into $Y ; L(X, Y)$ is a linear space.

Definition 6. (Simple Convergence Topology). This is the topology of the convergence at each point of $X$ and thus it is defined by the family of semi-norms of the form

$$
p(T)=\sup _{j=1, \ldots, r}\left\{q\left(T x_{j}\right)\right\}
$$

where $\left\{x_{j}\right\}_{j=1}^{r}$ are an arbitrary finite system of point of $X$ and $q$ an arbitrary continuous semi-norm on $Y . L(X, Y)$ endowed with this topology will be denoted by $L_{S}(X, Y)$ and it is a locally convex linear topological space.

Definition 7. (Bounded Convergence Topology). This is the topology of uniform convergence on bounded sets of X and thus it is defined by the family of semi-norms of the form

$$
p(T)=\sup _{x \in B}\{q(T x)\}
$$

where $B$ is an arbitrary bounded set of $X$ and $q$ an arbitrary continuous semi-norm on $Y . L(X, Y)$ endowed with this topology will be denoted by $L_{b}(X, Y)$ and it is a locally convex linear topological space.

Remark 2. Since any finite set of $X$ is bounded, the simple convergence topology is weaker than the bounded convergence topology.

\section{Bochner Integral}

In this section we will introduce the concept of integration of Bochner for vectorial functions in spaces of Fréchet. The properties here exposed are limited instead to the quasi-norm that induces the metric structure of the space of Fréchet.

Definition 8. Let $\mathfrak{F}$ be a complex Fréchet space and let I be an interval in $\mathbb{R} ;$ a function $f: I \rightarrow \mathfrak{F}$ is simple if it is of the form

$$
f(t)=\sum_{j=1}^{n} x_{j} \chi_{\Omega_{j}}(t)
$$

for some $n \geq 1, x_{j} \in \mathfrak{F}$ and Lebesgue measurable sets $\Omega_{j} \subset I$ with finite Lebesgue measure. In the representation of a simple function, the sets $\Omega_{j}$ may always be arranged to be disjoint, and then

$$
f(t)=\left\{\begin{array}{ll}
x_{j} & \text { for } t \in \Omega_{j}, j=1, \ldots, n \\
0 & \text { otherwise }
\end{array} .\right.
$$

Definition 9. Let $\mathfrak{F}$ be a complex Fréchet space and let I be an interval in $\mathbb{R} ;$ a function $f: I \rightarrow \mathfrak{F}$ is measurable if there is a sequence of simple functions $g_{h}$ such that

$$
f(t)=\mathfrak{F}-\lim _{h \rightarrow \infty} g_{h}(t)
$$

for almost all $t \in I$. 
Theorem 1. Let $\mathfrak{F}$ be a complex Fréchet space and let $I$ be an interval in $\mathbb{R} ;$ if $f: I \rightarrow \mathfrak{F}, g: I \rightarrow \mathfrak{F}, h: I \rightarrow \mathbb{C}$ are measurable then $f+g$ and $h f$ are measurable. Moreover, if $k: \mathfrak{F} \rightarrow Y$ is continuous, where $Y$ is a Fréchet space, then $k \circ f$ is measurable. In particular, $|f|_{\widetilde{\S}}$ is measurable.

Definition 10. Let $\mathfrak{F}$ be a complex Fréchet space and let I be an interval in $\mathbb{R}$; we say that $f: I \rightarrow \mathfrak{F}$ is countably valued if there is a countable partition $\left\{\Omega_{j}: j \in \mathbb{N}\right\}$ of I such that $f$ is constant on each $\Omega_{j}$.

Remark 3. $f$ is measurable if each $\Omega_{j}$ is measurable. Conversely, if $f$ is measurable then $\{t \in I: f(t)=x\}$ is measurable, where $x \in \mathfrak{F}$.

Definition 11. Let $\mathfrak{F}$ be a complex Fréchet space and let I be an interval in $\mathbb{R}$; we say that $f: I \rightarrow \mathfrak{F}$ is almost separably valued if there is a null set $\Omega_{0} \subset I$ such that $f\left(I-\Omega_{0}\right)$ is contained in a separable closed subspace of $\mathfrak{F}$.

Definition 12. Let $\mathfrak{F}$ be a complex Fréchet space and let I be an interval in $\mathbb{R}$; we say that $f: I \rightarrow \mathfrak{F}$ is weakly measurable if $x^{*} \circ f: t \rightarrow\left\langle f(t) \mid x^{*}\right\rangle$ is Lebesgue measurable for each $x^{*} \in \mathfrak{F}^{*}$.

Definition 13. For a simple function $g: I \rightarrow \mathfrak{F}, g(t)=\sum_{i=1}^{N} x_{i} \chi_{\Omega_{i}}(t)$, we define the Bochner's integral

$$
\int_{I} g(t) d t=\sum_{i=1}^{N} x_{i} \mathcal{L}^{1}\left(\Omega_{i}\right) .
$$

where $\mathcal{L}^{1}\left(\Omega_{i}\right)$ denote the Lebesgue measure of $\Omega_{i}$.

Definition 14. Let $\mathfrak{F}$ be a complex Fréchet space, I be an interval in $\mathbb{R}$ and $f: I \rightarrow \mathfrak{F}$ is weakly measurable; we say that $f$ is weakly integrable if exists $\int_{I}^{w^{*}} f(t) d t \in \mathfrak{F}$ such that

$$
\int_{I}\left\langle f(t) \mid x^{*}\right\rangle d t=\left\langle\int_{I}^{w^{*}} f(t) d t \mid x^{*}\right\rangle \quad \forall x^{*} \in \mathfrak{F}^{*}
$$

and the function $\left\langle f(t) \mid x^{*}\right\rangle$ is Lebesgue integrable $\forall x^{*} \in \mathfrak{F}^{*}$, i.e.,

$$
\int_{I}\left|\left\langle f(t) \mid x^{*}\right\rangle\right| d t<+\infty \quad \forall x^{*} \in \mathscr{F}^{*}
$$

Remark 4. If $g$ is a simple function then it is weakly integrable and the weak integral is the Bochner' integral of $g$.

Definition 15. Let $\mathfrak{F}$ be a complex Fréchet space and le I be an interval in $\mathbb{R} ; f: I \rightarrow \mathfrak{F}$ is called Bochner integrable if there exist a sequence of simple functions $\left\{g_{h}\right\}_{h \in \mathbb{N}}$ such that

$$
g_{h} \rightarrow f \quad \text { pointwise a.e. in I }
$$

and

$$
\lim _{h \rightarrow \infty} \int_{I}\left|f(t)-g_{h}(t)\right|_{\mathfrak{F}} d t=0 .
$$

If $f$ is Bochner integrable then the Bochner integral of $f$ on I is

$$
\int_{I} f(t) d t=\mathfrak{F}-\lim _{h \rightarrow \infty} \int_{I} g_{h}(t) d t .
$$

Theorem 2. Let $\mathfrak{F}$ be a complex Fréchet space and let I be an interval in $\mathbb{R} ; f: I \rightarrow \mathfrak{F}$ is Bochner integrable if and only if $f$ is measurable and $|f|_{\mathfrak{F}}$ is integrable. If $f$ is Bochner integrable then

$$
\left|\int_{I} f(t) d t\right|_{\mathscr{\gamma}} \leq \int_{I}|f(t)|_{\mathscr{F}} d t .
$$


Proof. If $f$ is Bochner integrable, then there exists an approximating sequence of simple functions $g_{n}$. Thus $f$ and $|f|_{\mathfrak{\mho}}$ are measurable. The integrability of $|f|_{\mathfrak{F}}$ follows from

$$
\int_{I}|f(t)|_{\mathscr{F}} d t \leq \int_{I}\left|g_{n}(t)\right|_{\mathfrak{F}} d t+\int_{I}\left|f(t)-g_{n}(t)\right|_{\mathfrak{F}} d t .
$$

Moreover,

$$
\begin{aligned}
\left|\int_{I} f(t) d t\right|_{\widetilde{F}} & =\lim _{n \rightarrow \infty}\left|\int_{I} g_{n}(t) d t\right|_{\mathscr{F}} \\
& \leq \lim _{n \rightarrow \infty} \int_{I}\left|g_{n}(t)\right|_{\mathfrak{F}} d t \\
& =\int_{I}|f(t)|_{\mathscr{F}} d t .
\end{aligned}
$$

To prove the converse statement, let $\left\{h_{n}\right\}_{n \in \mathbb{N}}$ be a sequence of simple functions approximating $f$ pointwise on $I-\Omega_{0}$, where $\left|\Omega_{0}\right|=0$.

Define simple functions by

$$
g_{n}(t)= \begin{cases}h_{n}(t) & \text { if }\left|h_{n}(t)\right|_{\mathfrak{F}} \leq|f(t)|_{\widetilde{F}}\left(1+n^{-1}\right), \\ 0 & \text { otherwise }\end{cases}
$$

Then

$$
\left|g_{n}(t)\right|_{\widetilde{F}} \leq|f(t)|_{\widetilde{F}}\left(1+n^{-1}\right)
$$

and

$$
\lim _{n \rightarrow \infty}\left|g_{n}(t)-f(t)\right|_{\mathfrak{r}}=0
$$

for all $t \in I-\Omega_{0}$.

Because the functions $|f|_{\mathfrak{\wp}}$ and $\left|g_{n}-f\right|_{\widetilde{\gamma}}$ are integrable and

$$
\left|g_{n}(t)-f(t)\right|_{\mathfrak{\wp}} \leq 3|f(t)|_{\mathfrak{\wp}},
$$

we can apply the scalar dominated convergenge theorem and obtain that

$$
\lim _{n \rightarrow \infty} \int_{I}\left|g_{n}(t)-f(t)\right|_{\mathfrak{F}} d t=0 .
$$

Remark 5. If $\mathcal{L}^{1}(I)<+\infty$ and $f$ is measurable then

$$
|f(t)|_{\mathfrak{\wp}}=\sum_{k=1}^{+\infty} \frac{\|f(t)\|_{k}}{2^{k}\left(1+\|f(t)\|_{k}\right)} \leq 1,
$$

thus $f$ is Bochner integrable.

Example 1. Let's take

$$
g(t)= \begin{cases}2 t \cos \left(\frac{\pi}{t^{2}}\right)+\frac{2 \pi}{t} \sin \left(\frac{\pi}{t^{2}}\right) & \text { if } 0<t \leq 1 \\ 0 & \text { if } t=0\end{cases}
$$

then we consider $f:[0,1] \rightarrow \mathfrak{F}$ define by

$$
f(t)=x g(t)
$$

where $x \in \mathfrak{F}$. We see that $f$ is Bochner integrable but

$$
\int_{0}^{1}\left|\left\langle f(t) \mid x^{*}\right\rangle\right| d t=\left|\left\langle x \mid x^{*}\right\rangle\right| \int_{0}^{1}|g(t)| d t=+\infty
$$

then $f$ is not weakly integrable. 
Example 2. Let $f:[0,+\infty) \rightarrow \mathfrak{F}$ define by

$$
f(t)= \begin{cases}x g(t) & \text { if } 0 \leq t \leq 1 \\ 0 & \text { if } t>1\end{cases}
$$

where $x \in \mathfrak{F}$; then $f$ is Bochner integrable but not weakly integrable.

Example 3. Let be $x: \mathbb{R} \rightarrow \mathbb{R}$ the function defined by $x(s)=1$ for all $s \in \mathbb{R}$, then $x \in L_{\text {loc }}^{2}(\mathbb{R})$. We define the semi-norms on $L_{l o c}^{2}(\mathbb{R}) b y$,

$$
\|z\|_{k}=\int_{B_{k}}|z(s)|^{2} d s
$$

where $\left\{B_{k}\right\}_{k \in \mathbb{N}}$ is a sequence of bounded closed subset of $\mathbb{R}$ such that $B_{k} \subset B_{k+1}$ and $\cup_{k \in \mathbb{N}} B_{k}=\mathbb{R}$. We take $B_{k}=$ $\left[-2^{2 k-1}, 2^{2 k-1}\right]$, then $\left|B_{k}\right|=2^{2 k}$. Let $f:[1,+\infty) \rightarrow L_{\text {loc }}^{2}(\mathbb{R})$ define by

$$
f(t)=\frac{1}{t^{2}} x
$$

then we have $\int_{1}^{+\infty}\left|\left\langle f(t) \mid x^{*}\right\rangle\right| d t=\left|\left\langle x \mid x^{*}\right\rangle\right|$ for all $x^{*} \in\left(L_{\text {loc }}^{2}(\mathbb{R})\right)^{*}$ and $\int_{1}^{+\infty}\left\langle f(t) \mid x^{*}\right\rangle d t=\left\langle x \mid x^{*}\right\rangle$ for all $x^{*} \in\left(L_{\text {loc }}^{2}(\mathbb{R})\right)^{*}$, then $f$ is weakly integrable but it is not Bochner integrable,

$$
\begin{aligned}
\int_{1}^{+\infty}|f(t)|_{F} d t & =\int_{1}^{+\infty} \sum_{k=1}^{+\infty} \frac{\|f(t)\|_{k}}{2^{k}\left(1+\|f(t)\|_{k}\right)} d t \\
& =\sum_{k=1}^{+\infty} \frac{1}{2^{k}} \int_{1}^{+\infty} \frac{\|f(t)\|_{k}}{\left(1+\|f(t)\|_{k}\right)} d t \\
& =\sum_{k=1}^{+\infty} \frac{1}{2^{k}} \int_{1}^{+\infty} \frac{\mid \frac{B_{k} \mid}{t^{2}}}{\left(1+\frac{\left|B_{k}\right|}{t^{2}}\right)} d t \\
& =\sum_{k=1}^{+\infty} \frac{2 \sqrt{\mid B_{k} k}}{2^{k}}\left[\frac{\pi}{2}-\arctan \left(\frac{1}{\sqrt[2]{\left|B_{k}\right|}}\right)\right] \\
& =\sum_{k=1}^{+\infty} \frac{\pi}{2}-\arctan \left(\frac{1}{2^{k}}\right)=+\infty
\end{aligned}
$$

We have the same results if we choose $B_{k}$ such that $\liminf _{k \rightarrow+\infty} \sqrt[2]{\frac{\left|B_{k+1}\right|}{\left|B_{k}\right|}}>2$.

Theorem 3. Let $f_{n}: I \rightarrow \mathfrak{F}$ be Bochner integrable functions. If

$$
\lim _{n \rightarrow \infty} f_{n}(t)=f(t)
$$

exists a.e. and if there exists an integrable function $g: I \rightarrow \mathbb{R}$ such that

$$
\left|f_{n}(t)\right|_{\mathfrak{r}} \leq g(t) \text { a.e. for all } n \in \mathbb{N},
$$

then $f$ is Bochner integrable and

$$
\lim _{n \rightarrow \infty} \int_{I} f_{n}(t) d t=\int_{I} f(t) d t .
$$

Moreover,

$$
\lim _{n \rightarrow \infty} \int_{I}\left|f_{n}(t)-f(t)\right|_{\mathfrak{F}} d t=0 .
$$

Proof. The function $f$ is Bochner integrable since it is measurable and since $|f|_{\mathfrak{F}}$ is integrable (because $|f|_{\mathfrak{F}} \leq g$ a.e.). We define

$$
h_{n}(t)=\left|f(t)-f_{n}(t)\right|_{\mathfrak{r}}
$$

for $t \in I$. Since $\left|h_{n}(t)\right|_{\widetilde{\gamma}} \leq 2 g(t)$ and $h_{n}(t) \rightarrow 0$ a.e., the scalar dominated convergence theorem implies that

$$
\lim _{n \rightarrow \infty} \int_{I}\left|f_{n}(t)-f(t)\right|_{\mathfrak{F}} d t=0 .
$$


By (2.27) we have

$$
\lim _{n \rightarrow \infty}\left|\int_{I} f_{n}(t) d t-\int_{I} f(t) d t\right|_{\mathscr{F}}=0
$$

\section{3. $\mathcal{L}_{B}(\mathfrak{F}, \mathbb{R})$-Weakly Integrable and $\mathfrak{F} s L^{p}(I, \mathfrak{F})$ Spaces}

To study the various relationships among the weak integral, definition (14) and the integral in Bochner sense, definition (15), we introduce a particular class of linear and continuous functionals. Then we will give a new definition of "weak" integral related to this class of linear and continuous functionals.

Definition 16. Let $\mathfrak{F}$ be a complex Fréchet space, then

$$
l \in \mathcal{L}_{B}(\mathfrak{F}, \mathbb{R})
$$

if $l$ is linear and there exists a constant $C_{l}$ such that

$$
|l(x)| \leq C_{l}|x|_{\mathfrak{F}} \quad \forall x \in \mathfrak{F} .
$$

Remark 6. $0 \in \mathcal{L}_{B}(\mathfrak{F}, \mathbb{R}) \neq \emptyset$.

Remark 7. $\mathcal{L}_{B}(\mathfrak{F}, \mathbb{R}) \subset \mathcal{L}(\mathfrak{F}, \mathbb{R})$.

Definition 17. Since $|x|_{\mathfrak{F}} \leq 1$ for all $x \in \mathfrak{F}$ we get $|l(x)| \leq C_{l}$ for all $x \in \mathfrak{F}$ and we define

$$
|l|_{\mathcal{L}_{B}(\mathfrak{r}, \mathbb{R})}=\sup _{x \in \mathfrak{F}}\{|l(x)|\} .
$$

Proposition 1. Let $\mathfrak{F}$ be a complex Fréchet space; then $|\cdot|_{\mathcal{L}_{B}(\mathfrak{r}, \mathbb{R})}$ is a quasi-norm and $\left(\mathcal{L}_{B}(\mathfrak{F}, \mathbb{R}),|\cdot|_{\mathcal{L}_{B}(\mathfrak{F}, \mathbb{R})}\right)$ is a quasinormed metric space.

Definition 18. Let $\mathfrak{F}$ a complex Fréchet space, I an interval in $\mathbb{R}$ and $\mathcal{L}_{B}(\mathfrak{F}, \mathbb{R}) \neq\{0\}$. Let $f: I \rightarrow \mathfrak{F}$ weakly measurable. We say that $f$ is $\mathcal{L}_{B}(\mathfrak{F}, \mathbb{R})$-weakly integrable if exists $\int_{I}^{w_{\mathcal{L}_{B}(\mathfrak{F}, \mathbb{R})}^{*}} f(t) d t \in \mathfrak{F}$ such that

$$
\int_{I}\left\langle f(t) \mid x^{*}\right\rangle d t=\left\langle\int_{I}^{w_{\mathcal{L}_{B}(\mathfrak{\mho}, \mathbb{R})}^{*}} f(t) d t \mid x^{*}\right\rangle \quad \forall x^{*} \in \mathcal{L}_{B}(\mathfrak{F}, \mathbb{R})
$$

and the function $\left\langle f(t) \mid x^{*}\right\rangle$ is Lebesgue integrable $\forall x^{*} \in \mathcal{L}_{B}(\mathfrak{F}, \mathbb{R})$, i.e.,

$$
\int_{I}\left|\left\langle f(t) \mid x^{*}\right\rangle\right| d t<+\infty \quad \forall x^{*} \in \mathcal{L}_{B}(\mathfrak{F}, \mathbb{R})
$$

Remark 8. If $f \in L^{1}(I, \mathfrak{F})$; then $f$ is $\mathcal{L}_{B}(\mathfrak{F}, \mathbb{R})$-weakly integrable.

Remark 9. If $f$ is weakly integrable; then $f$ is $\mathcal{L}_{B}(\mathfrak{F}, \mathbb{R})$-weakly integrable.

As a further comment we observe that it is possible to introduce other functional classes that have some connection with the integration.

Definition 19. Let $\left\{\|\cdot\|_{k}\right\}_{k \geq 1}$ be the the family of semi-norms that induces the topology on $\mathfrak{F}$ and $f: I \rightarrow \mathfrak{F}$ a Bochner measurable function; for $1 \leq p<\infty$ and for all $k \geq 1$, we define

$$
\|f\|_{k, L^{p}(I, \widetilde{Y})}=\left(\int_{I}\|f(t)\|_{k}^{p} d t\right)^{\frac{1}{p}}
$$

and

$$
L_{k}^{p}(I, \mathfrak{F})=\left\{f: I \rightarrow \mathfrak{F} \text { s.t. } f \text { is B-measurable and }\|f\|_{k, L^{p}(I, \mathfrak{F})}<\infty\right\}
$$


Definition 20. We define

$$
\mathfrak{F} s L^{p}(I, \mathfrak{F})=\bigcap_{k \geq 1} L_{k}^{p}(I, \mathfrak{F})
$$

Definition 21. Let $f \in \mathfrak{F} s L^{p}(I, \mathfrak{F})$; we define

$$
|f|_{\mathfrak{F} s L^{p}(I, \mathfrak{F})}=\sum_{k \geq 1} \frac{\|f\|_{k, L^{p}(I, \mathfrak{Y})}}{2^{k}\left(1+\|f\|_{k, L^{p}(I, \mathfrak{Y})}\right)} .
$$

Theorem 4. Sia $1 \leq p<\infty$; then $|\cdot|_{\widetilde{\mho} s L^{p}(I, \mathfrak{\mho})}$ si a quasi-norm and $\left(L^{p}(I, \mathfrak{F}),|\cdot|_{\mathfrak{\mho} s L^{p}(I, \mathfrak{F})}\right)$ is a metric space.

As a final observation we can say that here the considered spaces of Fréchet show a wide range of possible definitions of integral, each of which has interesting peculiarity. Substantially we will use the definition (14) but as shown in immediately the following proposition sometimes we will use the possible relationschip with (14), (15) and (18).

Definition 22. Let $\mathfrak{F}$ and $\mathbf{5}$ be a complex Fréchet space, then

$$
l \in \mathcal{L}_{B}(\mathfrak{F},(\mathfrak{5})
$$

if $l$ is linear and there exists a constant $C_{l}$ such that

$$
|l(x)|_{\mathfrak{5}} \leq C_{l}|x|_{\mathfrak{F}} \quad \forall x \in \mathfrak{F} .
$$

Remark 10. $0 \in \mathcal{L}_{B}(\mathfrak{F},(\mathfrak{5}) \neq \emptyset$.

Remark 11. $\mathcal{L}_{B}(\mathfrak{F},(\mathfrak{H}) \subset \mathcal{L}(\mathfrak{F},(\mathfrak{H})$.

Definition 23. Since $|x|_{\mathfrak{\Im}} \leq 1$ for all $x \in \mathfrak{F}$ we get $|l(x)|_{\mathfrak{5}} \leq C_{l}$ for all $x \in \mathfrak{F}$ and we define

$$
|l|_{\mathcal{L}_{B}(\mathfrak{F}, \mathfrak{F})}=\sup _{x \in \mathfrak{F}}\left\{|l(x)|_{\mathfrak{F}}\right\} .
$$

Remark 12. Let $\left(\mathfrak{5}=\mathfrak{F} \times \mathfrak{F}\right.$, with $|(x, y)|_{\mathfrak{5}}=|x|_{\mathfrak{F}}+|y|_{\mathfrak{F}}$, and $\pi: \mathfrak{5} \rightarrow \mathfrak{F}$ defined by

$$
\pi((x, y))=x
$$

or by

$$
\pi((x, y))=y
$$

then $\pi \in \mathcal{L}_{B}(\mathfrak{F},(\mathfrak{5})$.

Proposition 2. Let $T \in \mathcal{L}_{B}(\mathfrak{F},(\mathfrak{5})$ be a linear continuous operator between the Fréchet spaces $\mathfrak{F}$ and $\mathfrak{5}$. Let $f: I \rightarrow \mathfrak{F}$ be a Bochner integrable function; then $T \circ f: I \rightarrow \mathbb{G}$ defined by

$$
T \circ f(t)=T(f(t))
$$

is Bochner integrable and

$$
T \int_{I} f(t) d t=\int_{I} T(f(t)) d t .
$$

Proof. Since

$$
\int_{I}|T(f(t))|_{\mathfrak{G}} d t \leq \int_{I} C_{T}|f(t)|_{\mathfrak{F}_{\mathfrak{S}}} d t
$$

then $T \circ f: I \rightarrow \mathbb{5}$ is Bochner integrable. Moreover

$$
\int_{I} f(t) d t=\mathfrak{F}-\lim _{h \rightarrow \infty} \int_{I} g_{h}(t) d t
$$

and

$$
T\left(\int_{I} g_{h}(t) d t\right)=\int_{I} T\left(g_{h}(t)\right) d t
$$


since $T \in \mathcal{L}_{B}(\mathfrak{F},(\mathfrak{5})$ we have

$$
\begin{aligned}
T\left(\int_{I} f(t) d t\right) & =\mathfrak{5}-\lim _{h \rightarrow \infty} T\left(\int_{I} g_{h}(t) d t\right) \\
& =\left(\mathfrak{5}-\lim _{h \rightarrow \infty} \int_{I} T\left(g_{h}(t)\right) d t\right.
\end{aligned}
$$

and

$$
\int_{I}\left|T(f(t))-T\left(g_{h}(t)\right)\right|_{\mathfrak{G}} d t \leq \int_{I} C_{T}\left|f(t)-g_{h}(t)\right|_{\mathfrak{G}} d t ;
$$

then

$$
\left(\mathfrak{5}-\lim _{h \rightarrow \infty} \int_{I} T\left(g_{h}(t)\right) d t=\int_{I} T(f(t)) d t\right.
$$

and

$$
T\left(\int_{I} f(t) d t\right)=\int_{I} T(f(t)) d t .
$$

Proposition 3. Let $A: D(A) \rightarrow \mathfrak{F}$ be a closed linear operator on $\mathfrak{F}$. Let $f: I \rightarrow \mathfrak{F}$ be a Bochner integrable function such that $f(t) \in D(A)$ for all $t \in I$ and $A \circ f: I \rightarrow \mathfrak{F}$ is Bochner integrable; then $\int_{I} f(t) d t \in D(A)$ and

$$
A \int_{I} f(t) d t=\int_{I} A(f(t)) d t
$$

Proof. Let's take $\mathfrak{F} \times \mathfrak{F}$ with the quasi-norm

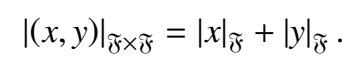

The graph $G(A)$ of $A$ is a closed subspace of $\mathfrak{F} \times \mathfrak{F}$. Define $g: I \rightarrow G(A) \subset \mathfrak{F} \times \mathfrak{F}$ by

$$
g(t)=(f(t), A(f(t)))
$$

then

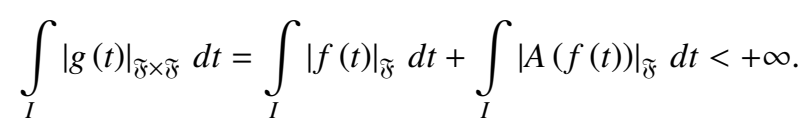

By theorem (2) $g$ is Bochner integrable, moreover

$$
\int_{I} g(t) d t \in G(A)
$$

and applying proposition (2) to the two projection maps of $\mathfrak{F} \times \mathfrak{F}$ onto $\mathfrak{F}$ shows that

$$
\int_{I} g(t) d t=\left(\int_{I} f(t) d t, \int_{I} A(f(t)) d t\right),
$$

this give the result.

\section{Laplace's Integrals on Fréchet Spaces}

Definition 24. Let $\mathfrak{F}$ a Fréchet space and $f \in L_{l o c}^{1}([0,+\infty)$, $)$; then

$$
\widehat{f}(\lambda)=\int_{0}^{+\infty} e^{-\lambda t} f(t) d t=\lim _{\tau \rightarrow+\infty} \int_{0}^{\tau} e^{-\lambda t} f(t) d t
$$

is the Laplace integral of $f$. 
Definition 25. Let $\mathfrak{F}$ a Fréchet space, $f \in L_{\text {loc }}^{1}([0,+\infty)$, $\mathfrak{F})$ and $\widehat{f}$ the Laplace integral of $f$; then

$$
a b s(f)=\inf \{\operatorname{Re}\{\lambda\}: \hat{f}(\lambda) \text { exists }\}
$$

is the abscissa of convergence of $\widehat{f}$.

Proposition 4. Let $\mathfrak{F}$ a Fréchet space and $f \in L_{l o c}^{1}([0,+\infty), \mathfrak{F})$; then the Laplace integral of $f, \hat{f}(\lambda)$, converge if $\mathfrak{R}\{\lambda\}>a b s(f)$ and not converge if $\operatorname{Re}\{\lambda\}<a b s(f)$.

Proof. $\widehat{f}(\lambda)$ does not exist if $\operatorname{Re}\{\lambda\}<a b s(f)$.

Let $\lambda_{0} \in \mathbb{C}$ and $a b s(f)<R e \lambda_{0}$, define

$$
G_{0}(\tau)=\int_{0}^{\tau} e^{-\lambda_{0} s} f(s) d s
$$

if $\widehat{f}\left(\lambda_{0}\right)$ exists then

$$
\widehat{f}\left(\lambda_{0}\right)=\widetilde{F}-\lim _{\tau \rightarrow+\infty} G_{0}(\tau)
$$

and

$$
\left|G_{0}(\tau)\right|_{\mathscr{\mho}} \leq C_{1} \quad \forall \tau \in[0,+\infty)
$$

moreover we have

$$
\left\|G_{0}(\tau)\right\|_{k} \leq\left\|G_{0}(\tau)-\widehat{f}\left(\lambda_{0}\right)\right\|_{k}+\left\|\widehat{f}\left(\lambda_{0}\right)\right\|_{k} \leq C_{2}
$$

for every $k \in \mathbb{N}$ and $\tau \rightarrow+\infty$; then

$$
\left\|G_{0}(\tau)\right\|_{k} \leq C_{3} \quad \forall \tau \in[0,+\infty), \forall k \in \mathbb{N} .
$$

Let $\int_{0}^{\tau} e^{-\lambda s} f(s) d s$, integration by parts gives

$$
\int_{0}^{\tau} e^{-\lambda s} f(s) d s=e^{-\left(\lambda-\lambda_{0}\right) \tau} G_{0}(\tau)+\left(\lambda-\lambda_{0}\right) \int_{0}^{\tau} e^{-\left(\lambda-\lambda_{0}\right) s} G_{0}(s) d s
$$

and we must proof that

$$
\mathfrak{F}-\lim _{\tau \rightarrow+\infty} \int_{0}^{\tau} e^{-\lambda s} f(s) d s
$$

exist if $\operatorname{Re}\{\lambda\}>\operatorname{Re}\left\{\lambda_{0}\right\}$.

Let $\left(\lambda-\lambda_{0}\right) \int_{\tau_{1}}^{\tau_{2}} e^{-\left(\lambda-\lambda_{0}\right) s} G_{0}(s) d s ;$ then by (4.7)

$$
\begin{aligned}
\left\|\left(\lambda-\lambda_{0}\right) \int_{\tau_{1}}^{\tau_{2}} e^{-\left(\lambda-\lambda_{0}\right) s} G_{0}(s) d s\right\|_{k} & \leq\left|\lambda-\lambda_{0}\right| \int_{\tau_{1}}^{\tau_{2}} e^{-\left(\operatorname{Re}\{\lambda\}-\operatorname{Re}\left\{\lambda_{0}\right\}\right) s}\left\|G_{0}(s)\right\|_{k} d s \\
& \leq\left|\lambda-\lambda_{0}\right| C_{3} \int_{\tau_{1}}^{\tau_{2}} e^{-\left(\operatorname{Re}\{\lambda\}-\operatorname{Re}\left\{\lambda_{0}\right\}\right) s} d s \rightarrow 0 \quad \text { for } \tau \rightarrow+\infty, \forall k \in \mathbb{N},
\end{aligned}
$$

if $\operatorname{Re}\{\lambda\}>\operatorname{Re}\left\{\lambda_{0}\right\}$.

From (4.10) we have

$$
\mathfrak{F}-\lim _{\tau \rightarrow+\infty} \int_{0}^{\tau}\left(\lambda-\lambda_{0}\right) e^{-\left(\lambda-\lambda_{0}\right) s} G_{0}(s) d s=\int_{0}^{+\infty}\left(\lambda-\lambda_{0}\right) e^{-\left(\lambda-\lambda_{0}\right) s} G_{0}(s) d s .
$$

if $\operatorname{Re}\{\lambda\}>\operatorname{Re}\left\{\lambda_{0}\right\}$; then the (4.11) gives

$$
\widehat{f}(\lambda)=\widetilde{F}-\lim _{\tau \rightarrow+\infty} \int_{0}^{\tau} e^{-\lambda s} f(s) d s=\int_{0}^{+\infty}\left(\lambda-\lambda_{0}\right) e^{-\left(\lambda-\lambda_{0}\right) s} G_{0}(s) d s .
$$


Definition 26. For $f:[0,+\infty) \rightarrow \mathfrak{F}$ the exponential growth bound is given by

$$
\omega(f)=\inf \left\{w \in \mathbb{R}:\left\{e^{-w t} f(t): t \geq 0\right\} \text { is bounded in } \mathfrak{F}\right\} .
$$

Remark 13. $a b s(f) \leq \omega(f)$.

Proof. Let $\lambda>\sigma>\omega(f)$ then exist $C_{k}>0$ such that

$$
\left\|e^{-\sigma t} f(t)\right\|_{k} \leq C_{k} \quad \forall t \geq 0, \forall k \in \mathbb{N} .
$$

We have

$$
\left\|\int_{t_{1}}^{t_{2}} e^{-\lambda t} f(t) d t\right\|_{k} \leq \int_{t_{1}}^{t_{2}} e^{-(\lambda-\sigma) t}\left\|e^{-\sigma t} f(t)\right\|_{k} d t
$$

then

$$
\left\|\int_{t_{1}}^{t_{2}} e^{-\lambda t} f(t) d t\right\|_{k} \leq C_{k} \int_{t_{1}}^{t_{2}} e^{-(\lambda-\sigma) t} d t \rightarrow 0
$$

for $t_{1}, t_{2} \rightarrow+\infty$ and $\lambda>\sigma>\omega(f)$, thus $\widehat{f}(\lambda)=\mathfrak{F}-\lim _{\tau \rightarrow+\infty} \int_{0}^{\tau} e^{-\lambda s} f(s) d s$ exists and $a b s(f) \leq \omega(f)$.

Definition 27. Let $F(t)=\int_{0}^{t} f(s) d s$, where $f \in L_{l o c}^{1}([0,+\infty)$, $\mathfrak{F})$, we define

$$
F_{\infty}= \begin{cases}\mathfrak{J}-\lim _{t \rightarrow+\infty} \int_{0}^{t} f(s) d s & \text { if the limit exists, } \\ 0 & \text { otherwise. }\end{cases}
$$

Lemma 1. Let $\mathfrak{F}$ a Fréchet space and $f \in L_{l o c}^{1}([0,+\infty)$, $)$; then

$$
\operatorname{abs}(f)=\omega\left(F-F_{\infty}\right) .
$$

Proof. (Step 1)

Let $a b s(f)<+\infty, \lambda_{0}>a b s(f)$,

$$
G_{0}(t)=\int_{0}^{t} e^{-\lambda_{0} s} f(s) d s
$$

and

$$
\mathfrak{F}-\lim _{t \rightarrow+\infty} G_{0}(t)=\int_{0}^{+\infty} e^{-\lambda_{0} s} f(s) d s=\widehat{f}\left(\lambda_{0}\right) \in \mathfrak{F}
$$

then for all $k \in \mathbb{N}$

$$
\left\|G_{0}(t)\right\|_{k} \leq C_{k} \quad \forall t \geq 0
$$

Let $a b s(f)>0$ then $F_{\infty}=0$ and, for $\lambda_{0}>a b s(f)$, integration by parts gives

$$
\int_{0}^{t} e^{\lambda_{0} s} e^{-\lambda_{0} s} f(s) d s=e^{\lambda_{0} t} G_{0}(t)-\lambda_{0} \int_{0}^{t} e^{\lambda_{0} s} G_{0}(s) d s
$$

then for all $k \in \mathbb{N}$

$$
\|F(t)\|_{k} \leq 2 C_{k} e^{\lambda_{0} t} \quad \forall t \geq 0 .
$$

The set $\left\{F(t) e^{-\lambda_{0} t}: t \geq 0\right\}$ is bounded in $\mathfrak{F}$ and $\varpi\left(F-F_{\infty}\right)<\lambda_{0}$ then $\varpi\left(F-F_{\infty}\right) \leq a b s(f)$. Let $a b s(f)=0$ then if $F_{\infty}=0$ the same procedure as above yields $\varpi\left(F-F_{\infty}\right) \leq a b s(f)$. 
If $F_{\infty}=\mathfrak{F}-\lim _{t \rightarrow+\infty} F(t)$ then for all $k \in \mathbb{N}\left\|F(t)-F_{\infty}\right\| \leq C_{k}$ for all $t \geq 0$, thus the set $\left\{F(t)-F_{\infty}: t \geq 0\right\}$ is bounded in $\mathfrak{F}$ and $\varpi\left(F-F_{\infty}\right) \leq 0=a b s(f)$.

If $a b s(f)<0$ choose $a b s(f)<\lambda_{0}<0$ then

$$
\begin{aligned}
F(r)-F(s) & =\int_{s}^{r} e^{\lambda_{0} t} f(t) e^{-\lambda_{0} t} d t \\
& =e^{\lambda_{0} r} G_{0}(r)-e^{\lambda_{0} s} G_{0}(s)-\lambda_{0} \int_{s}^{r} e^{\lambda_{0} t} G_{0}(t) d t
\end{aligned}
$$

and for all $k \in \mathbb{N}$

$$
\begin{aligned}
\left\|F(s)-F_{\infty}\right\|_{k} & =\left\|e^{\lambda_{0} s} G_{0}(s)+\lambda_{0} \int_{s}^{+\infty} e^{\lambda_{0} t} G_{0}(t) d t\right\|_{k} \\
& \leq 2 C_{k} e^{\lambda_{0} s}
\end{aligned}
$$

for all $s \geq 0$; thus the set $\left\{\left(F(t)-F_{\infty}\right) e^{-\lambda_{0} t}: t \geq 0\right\}$ is bounded in $\mathfrak{F}$ and $\varpi\left(F-F_{\infty}\right)<\lambda_{0}$ then $\varpi\left(F-F_{\infty}\right) \leq a b s(f)$.

(Step 2)

Suppose that $\varpi\left(F-F_{\infty}\right)<+\infty$ and let $\varpi\left(F-F_{\infty}\right)<w$; since $F$ is continuous, there exist $M_{k}$ such that $\left\|F(t)-F_{\infty}\right\|_{k} \leq$ $M_{k} e^{w t}$ for all $t \geq 0$.

Let $\varpi\left(F-F_{\infty}\right)<w<\lambda$, using the fact that $F-F_{\infty}$ is an antiderivative of $f$, integration by parts gives

$$
\int_{0}^{t} e^{-\lambda s} f(s) d s=e^{-\lambda t}\left(F(t)-F_{\infty}\right)+F_{\infty}+\lambda \int_{0}^{t} e^{-\lambda s}\left(F(s)-F_{\infty}\right) d s
$$

then $\widehat{f}(\lambda)$ exists,

$$
\widehat{f}(\lambda)=F_{\infty}+\lambda\left(\widehat{F-F_{\infty}}\right)(\lambda)
$$

and $a b s(f) \leq \varpi\left(F-F_{\infty}\right)$.

Remark 14. If $w \geq 0$; then the triangle inequality implies that $\varpi(F) \leq w$ if and only if $\varpi\left(F-F_{\infty}\right) \leq w$. Thus $f$ is Laplace transformable if and only if $F(t)=\int_{0}^{t} f(s) d s$ is exponentially bounded and abs $(f) \leq w \Leftrightarrow \varpi(F) \leq w$.

Theorem 5. Let $f \in L_{\text {loc }}^{1}([0,+\infty)$, $)$ with abs $(f)<\infty$. Then

$$
\lambda \mapsto \widehat{f}(\lambda)
$$

is holomorphic for $\operatorname{Re}\{\lambda\}>$ abs $(f)$ and, for all $n \in \mathbb{N}$ and $\operatorname{Re}\{\lambda\}>$ abs $(f)$,

$$
\widehat{f}^{(n)}(\lambda)=\int_{0}^{+\infty} e^{-\lambda t}(-t)^{n} f(t) d t
$$

as an improper Bochner integral.

Proof. Let's define $q_{h}: \mathbb{C} \rightarrow F$ for every $h \in \mathbb{N}$ by

$$
q_{h}(\lambda)=\int_{0}^{h} e^{-\lambda t} f(t) d t
$$

and $q_{h, j}: \mathbb{C} \rightarrow F$ for every $h, j \in \mathbb{N}$ by

$$
q_{h, j}(\lambda)=\sum_{n=1}^{j} \frac{\lambda^{n}}{n !} \int_{0}^{h}(-t)^{n} f(t) d t
$$


We see that for every $k \in \mathbb{N}$ and $j>i$

$$
\begin{aligned}
\left\|q_{h, j}(\lambda)-q_{h, i}(\lambda)\right\|_{k} & \leq \sum_{n=i}^{j} \frac{|\lambda|^{n}}{n !}\left\|\int_{0}^{h} t^{n} f(t) d t\right\|_{k} \\
& \leq \sum_{n=i}^{j} \frac{|\lambda h|^{n}}{n !}\left\|\int_{0}^{h} f(t) d t\right\|_{k} \\
& \leq C_{k} \sum_{n=i}^{j} \frac{|\lambda h|^{n}}{n !} .
\end{aligned}
$$

Fix $\varepsilon>0$ then exists $j_{k, \varepsilon} \in \mathbb{N}$ such that $\left\|q_{h, j}(\lambda)-q_{h, i}(\lambda)\right\|_{k}<\varepsilon$ for all $i, j>j_{k, \varepsilon}$ then we have

$$
q_{h}(\lambda)=\widetilde{r}-\lim _{j \rightarrow+\infty} q_{h, j}(\lambda) .
$$

The limits exist uniformly for $\lambda$ in a bounded subset of $\mathbb{C}$. By the Weiesrtass convergence theorem, the functions $q_{h}$ are entire and $q_{h}^{(j)}(\lambda)=\int_{0}^{h} e^{-\lambda t}(-t)^{j} f(t) d t$ for all $j=1,2, \ldots$

Let $\lambda_{0} \in \mathbb{C}, a b s(f)<\operatorname{Re} \lambda_{0}$ and

$$
G_{0}(\tau)=\int_{0}^{\tau} e^{-\lambda_{0} s} f(s) d s
$$

$\widehat{f}\left(\lambda_{0}\right)$ exists then

$$
\widehat{f}\left(\lambda_{0}\right)=\mathfrak{F}-\lim _{\tau \rightarrow+\infty} G_{0}(\tau)
$$

and

$$
\left|G_{0}(\tau)\right|_{\mathfrak{\mho}} \leq C_{1} \quad \forall \tau \in[0,+\infty)
$$

moreover we have

$$
\left\|G_{0}(\tau)\right\|_{k} \leq\left\|G_{0}(\tau)-\widehat{f}\left(\lambda_{0}\right)\right\|_{k}+\left\|\widehat{f}\left(\lambda_{0}\right)\right\|_{k} \leq C_{2}
$$

for every $k \in \mathbb{N}$ and $\tau \rightarrow+\infty$; then

$$
\left\|G_{0}(\tau)\right\|_{k} \leq C_{3} \quad \forall \tau \in[0,+\infty), \forall k \in \mathbb{N}
$$

Let $\left(\lambda-\lambda_{0}\right) \int_{\tau_{1}}^{\tau_{2}} e^{-\left(\lambda-\lambda_{0}\right) s} G_{0}(s) d s$; then

$$
\begin{aligned}
\left\|\left(\lambda-\lambda_{0}\right) \int_{\tau_{1}}^{\tau_{2}} e^{-\left(\lambda-\lambda_{0}\right) s} G_{0}(s) d s\right\|_{k} & \leq\left|\lambda-\lambda_{0}\right| \int_{\tau_{1}}^{\tau_{2}} e^{-\left(\operatorname{Re}\{\lambda\}-\operatorname{Re}\left\{\lambda_{0}\right\}\right) s}\left\|G_{0}(s)\right\|_{k} d s \\
& \leq\left|\lambda-\lambda_{0}\right| C_{3} \int_{\tau_{1}}^{\tau_{2}} e^{-\left(\operatorname{Re}\{\lambda\}-\operatorname{Re}\left\{\lambda_{0}\right\}\right) s} d s \rightarrow 0 \quad \text { for } \tau \rightarrow+\infty, \forall k \in \mathbb{N},
\end{aligned}
$$

if $\operatorname{Re}\{\lambda\}>\operatorname{Re}\left\{\lambda_{0}\right\}$ and we have

$$
\mathfrak{F}-\lim _{\tau \rightarrow+\infty} \int_{0}^{\tau}\left(\lambda-\lambda_{0}\right) e^{-\left(\lambda-\lambda_{0}\right) s} G_{0}(s) d s=\int_{0}^{+\infty}\left(\lambda-\lambda_{0}\right) e^{-\left(\lambda-\lambda_{0}\right) s} G_{0}(s) d s .
$$

if $\operatorname{Re}\{\lambda\}>\operatorname{Re}\left\{\lambda_{0}\right\}$. Let $\int_{0}^{\tau} e^{-\lambda_{0} s} f(s) d s$; integration by parts gives

$$
\begin{aligned}
\widehat{f}(\lambda)-q_{h}(\lambda) & =\int_{h}^{+\infty} e^{-\left(\lambda-\lambda_{0}\right) t} e^{-\lambda_{0} t} f(t) d t \\
& =-e^{-\left(\lambda-\lambda_{0}\right) h} G_{0}(h)+\left(\lambda-\lambda_{0}\right) \int_{h}^{+\infty} e^{-\left(\lambda-\lambda_{0}\right) t} G_{0}(t) d t .
\end{aligned}
$$

It follows that $q_{h}$ converges to $\widehat{f}$ uniformly on compact subset of $\{\lambda: \operatorname{Re}\{\lambda\}>a b s(f)\}$. By the Weiestrass convergence theorem, $\widehat{f}$ is holomorphic and $q_{h}^{(m)}(\lambda) \rightarrow \widehat{f}^{(m)}(\lambda)$ as $h \rightarrow+\infty$, for $\operatorname{Re}\{\lambda\}>a b s(f)$. 
Proposition 5. Let $f \in L_{l o c}^{1}([0,+\infty), \mathfrak{F}), \mu \in \mathbb{C}$ and $s \in[0,+\infty)$. Let

$$
\begin{aligned}
& g(t)=e^{-\mu t} f(t) \text { for } t \geq 0, \\
& f_{s}(t)=f(s+t) \text { for } t \geq 0, \\
& h_{s}(t)=\left\{\begin{array}{l}
f(t-s) \text { for } t \geq s, \\
0 \text { for } o \leq t<s .
\end{array}\right.
\end{aligned}
$$

Let $\lambda \in \mathbb{C}$; then

$$
\begin{array}{ll}
\widehat{g}(\lambda) \text { exists } \Leftrightarrow \widehat{f}(\lambda+\mu) \text { exists; } & \text { if } \widehat{g}(\lambda) \text { exists then } \widehat{g}(\lambda)=\widehat{f}(\lambda+\mu) . \\
\widehat{f_{s}}(\lambda) \text { exists } \Leftrightarrow \widehat{f}(\lambda) \text { exists; } & \text { if } \widehat{f_{s}}(\lambda) \text { exists then } \widehat{f_{s}}(\lambda)=e^{\lambda s}\left(\widehat{f}(\lambda)-\int_{0}^{s} e^{-\lambda t} f(t) d t\right) . \\
\widehat{h_{s}}(\lambda) \text { exists } \Leftrightarrow \widehat{f}(\lambda) \text { exists; } & \text { if } \widehat{h_{s}}(\lambda) \text { exists then } \widehat{h_{s}}(\lambda)=e^{-\lambda s} \widehat{f}(\lambda) .
\end{array}
$$

Proof. Results follow from the formulae:

$$
\begin{aligned}
\int_{0}^{\tau} e^{-\lambda t} g(t) d t & =\int_{0}^{\tau} e^{-(\lambda+\mu) t} f(t) d t \\
\int_{0}^{\tau} e^{-\lambda t} f_{s}(t) d t & =e^{\lambda s}\left(\int_{0}^{s+\tau} e^{-\lambda t} f(t) d t-\int_{0}^{s} e^{-\lambda t} f(t) d t\right) \\
\int_{0}^{\tau} e^{-\lambda t} h_{s}(t) d t & =e^{-\lambda s} \int_{0}^{\tau-s} e^{-\lambda t} f(t) d t \quad(\tau>s) .
\end{aligned}
$$

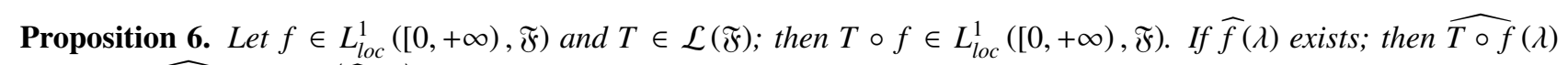
exists and $\widehat{T \circ f}(\lambda)=T(\widehat{f}(\lambda))$.

Proof. By proposition (2), $T \circ f \in L_{l o c}^{1}([0,+\infty), \mathfrak{F})$ and

$$
\int_{0}^{\tau} e^{-\lambda t}(T \circ f)(t) d t=T \int_{0}^{\tau} e^{-\lambda t} f(t) d t .
$$

The second statement follows from (4.19) letting $\tau \rightarrow+\infty$.

Proposition 7. Let $f \in L_{l o c}^{1}([0,+\infty)$, $\mathfrak{F})$ and $A$ a closed operator on $\mathfrak{F}$; suppose that $f(t) \in D(A)$ for a.e. $t \in[0,+\infty)$ and $A \circ f \in L_{l o c}^{1}([0,+\infty)$, 宁). If $\widehat{f}(\lambda)$ and $\widehat{A \circ f}(\lambda)$ exist, then $\widehat{f}(\lambda) \in D(A)$ and $\widehat{A \circ f}(\lambda)=A(\widehat{f}(\lambda))$.

Proof. By proposition (3),

$$
\int_{0}^{\tau} e^{-\lambda t}(A \circ f)(t) d t=A \int_{0}^{\tau} e^{-\lambda t} f(t) d t .
$$

Since $A$ is closed the second statement follows from (4.20) letting $\tau \rightarrow+\infty$.

Lemma 2. Let $a, b>0$, define $\lambda_{n}=a+n b$ and $e_{-\lambda_{n}}(t)=e^{-\lambda_{n} t}$; then $\left\{e_{-\lambda_{n}}: n \in \mathbb{N} \backslash\{0\}\right\}$ is total in $L^{1}([0,+\infty))$.

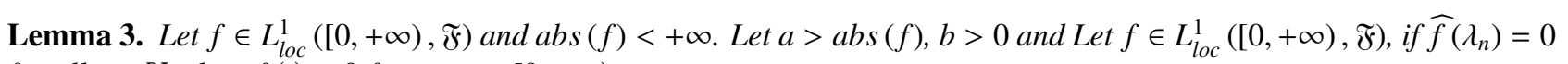
for all $n \in \mathbb{N}$; then $f(t)=0$ for a.e. $t \in[0,+\infty)$.

Proof. We can assume that $a>\max \{a b s(f), 0\}$. Define $F(t)=\int_{0}^{t} f(s) d s$, for $t \geq 0$; then $0=\widehat{f}\left(\lambda_{n}\right)=\lambda_{n} \widehat{F}\left(\lambda_{n}\right)$ for all $n \in \mathbb{N}$. By remark (14) we have $a>\omega(F)$, thus $G(t)=e^{-a t} F(t)$ is continuous and $\|G(t)\|_{k}<M_{k}$ for $t>0$, moreover we have $\widehat{G}(n b)=\widehat{F}(a+n b)=0$. Let $x^{*} \in \widetilde{F}^{*}$ we define $g_{x^{*}}(t)=\left\langle G(t) \mid x^{*}\right\rangle$, then we have $g_{x^{*}} \in L^{\infty}([0,+\infty))=\left(L^{1}([0,+\infty))\right)^{*}$ and

$$
\left\langle e^{-n b} \mid g_{x^{*}}\right\rangle=\left\langle\widehat{G}(n b) \mid x^{*}\right\rangle=0 .
$$

Since $\left\{e^{-n b}: n \in \mathbb{N}\right\}$ is a total subset of $L^{1}([0,+\infty))$ then by lemma (2) $g_{x^{*}}(t)=0$ for all $t \geq 0$ and $x^{*} \in \mathfrak{F}^{*}$; this implies that $F(t)=0$ for all $t \geq 0$ and thus $f(t)=0$ a.e.. 
Theorem 6. Let $f, g \in L_{l o c}^{1}([0,+\infty)$, $\mathfrak{F})$ and abs $(f)$, abs $(g)<+\infty$. Let $\lambda_{0}>\max \{a b s(f)$, abs $(g)\}$, and suppose that $\widehat{f}(\lambda)=\widehat{g}(\lambda)$ for $\lambda \geq \lambda_{0}$ then $f(t)=g(t)$ for a.e. $t \in[0,+\infty)$.

Proof. The proof follows from lemma (3).

Theorem 7. Let $f \in L_{l o c}^{1}([0,+\infty)$, $\mathfrak{F})$, suppose that abs $(f)<+\infty$ and that $t>0$ is a Lebesgue point of $f$; then

$$
f(t)=\mathscr{F}-\lim _{k \rightarrow \infty}(-1)^{k} \frac{1}{k !}\left(\frac{k}{t}\right)^{k+1} \widehat{f}^{(k)}\left(\frac{k}{t}\right)
$$

Proof. Let $f \in L_{\text {loc }}^{1}([0,+\infty), \mathfrak{F})$ and $\max \{a b s(f), 0\}<w<+\infty$. By remark (14) $w>\varpi(F)$, where $F(t)=\int_{0}^{t} f(s) d s$. Let $t>0$ be a Lebesgue point of $f, n \in \mathbb{N}$ such that $n>w t$; let

$$
G(s)=\int_{t}^{s} f(r)-f(t) d r=F(s)-F(t)-f(t)(s-t) \quad s \geq t \geq 0 .
$$

Since $w>\varpi(F)$, then the set $\left\{e^{-w s} G(s): s \geq 0\right\}$ is bounded in $\mathfrak{F}$ and there exists a sequence of positive real numbers, $\left\{M_{k}\right\}_{k \in \mathbb{N}}$, such that $\left\|e^{-w s} G(s)\right\|_{k} \leq M_{k}$ for all $s \geq 0$. Since

$$
\int_{0}^{+\infty} e^{-t} t d t=\left[-e^{-t} t\right]_{0}^{+\infty}+\int_{0}^{+\infty} e^{-t} d t=\left[-e^{-t}\right]_{0}^{+\infty}=1
$$

and

moreover

$$
\int_{0}^{+\infty} e^{-t} t^{2} d t=\left[-e^{-t} t^{2}\right]_{0}^{+\infty}+2 \int_{0}^{+\infty} e^{-t} t d t=2
$$

$$
\int_{0}^{+\infty} e^{-t} t^{3} d t=\left[-e^{-t} t^{3}\right]_{0}^{+\infty}+3 \int_{0}^{+\infty} e^{-t} t^{2} d t=3 !
$$

by induction,

$$
\int_{0}^{+\infty} e^{-t} t^{n} d t=n !
$$

if we put $t=\lambda s$, where $\lambda>0$, we have

$$
\lambda^{n+1} \int_{0}^{+\infty} e^{-\lambda s} s^{n} d s=n !
$$

then

$$
\frac{\lambda^{n+1}}{n !} \int_{0}^{+\infty} e^{-\lambda s} s^{n} d s=1
$$

for all $\lambda>0$. By theorem (5) we have

$$
\widehat{f}^{(n)}(\lambda)=\int_{0}^{+\infty} e^{-\lambda s}(-s)^{n} f(s) d s
$$

then

$$
(-1)^{n} \frac{1}{n !}\left(\frac{n}{t}\right)^{n+1} \widehat{f}^{(n)}\left(\frac{n}{t}\right)=\frac{1}{n !}\left(\frac{n}{t}\right)^{n+1} \int_{0}^{+\infty} e^{-\frac{n}{t} s} s^{n} f(s) d s
$$

moreover

$$
f(t)=\frac{\lambda^{n+1}}{n !} \int_{0}^{+\infty} e^{-\lambda s} s^{n} f(t) d s
$$


put $\lambda=\frac{n}{t}$ we have

$$
f(t)=\frac{1}{n !}\left(\frac{n}{t}\right)^{n+1} \int_{0}^{+\infty} e^{-\frac{n}{t} s} s^{n} f(t) d s
$$

Let

$$
J_{n}=(-1)^{n} \frac{1}{n !}\left(\frac{n}{t}\right)^{n+1} \widehat{f}^{(n)}\left(\frac{n}{t}\right)-f(t)
$$

then

$$
J_{n}=\frac{1}{n !}\left(\frac{n}{t}\right)^{n+1} \int_{0}^{+\infty} e^{-\frac{n}{t} s} s^{n}(f(s)-f(t)) d s
$$

let

$$
G(s)=\int_{t}^{s} f(r)-f(t) d r=F(s)-F(t)-f(t)(s-t) \quad s \geq t \geq 0
$$

thus by (4.22) we get

$$
J_{n}=\frac{1}{n !}\left(\frac{n}{t}\right)^{n+1}\left(\left[\left(e^{-\frac{n}{t} s} s^{n}\right) G(s)\right]_{0}^{+\infty}+n \int_{0}^{+\infty} e^{-\frac{n}{t} s} s^{n-1}\left(\frac{s}{t}-1\right) G(s) d s\right) .
$$

Let's take $\left[\left(e^{-\frac{n}{t} s} s^{n}\right) G(s)\right]_{0}^{+\infty}$; then

$$
\left(e^{-\frac{n}{t} s} s^{n}\right) G(s)=\left(e^{-\frac{n}{t} s} s^{n}\right) \int_{t}^{s} f(r)-f(t) d r=\left(e^{-\frac{n}{t} s} s^{n}\right)(F(s)-F(t)-f(t)(s-t))
$$

moreover, since $n>w t$, we have

$$
\mathfrak{F}-\lim _{s \rightarrow+\infty}\left(e^{-\frac{n}{t} s} s^{n}\right) G(s)=0
$$

and

$$
J_{n}=\frac{1}{n !}\left(\frac{n}{t}\right)^{n+1} n \int_{0}^{+\infty} e^{-\frac{n}{t} s} s^{n-1}\left(\frac{s}{t}-1\right) G(s) d s
$$

If $\frac{s}{t}=u$; then

$$
\begin{aligned}
J_{n} & =\frac{1}{n !}\left(\frac{n}{t}\right)^{n+1} n \int_{0}^{+\infty} e^{-\frac{n}{t} s} s^{n-1}\left(\frac{s}{t}-1\right) G(s) d s \\
& =\frac{n^{n+2}}{n ! t} \int_{0}^{+\infty} e^{-\frac{n}{t} s} \frac{1}{t^{n-1}} s^{n-1}\left(\frac{s}{t}-1\right) G(s) \frac{1}{t} d s \\
& =\frac{n^{n+2}}{n ! t} \int_{0}^{+\infty} e^{-n u} u^{n-1}(u-1) G(t u) d u .
\end{aligned}
$$

Let $\varepsilon>0$; since $t$ is a Lebesgue point of $f$, there exists $0<\delta<1$ such that

$$
\frac{1}{t}\|G(u t)\|_{k}=\left\|\frac{1}{t} \int_{t}^{u t} f(r)-f(t) d r\right\|_{r} \leq \frac{\varepsilon}{3}|u-1|
$$

if $|u-1| \leq \delta$.

Let's define

$$
J_{1, n}=\frac{n^{n+2}}{n ! t} \int_{1-\delta}^{1+\delta} e^{-n u} u^{n-1}(u-1) G(t u) d u
$$


then

$$
\begin{aligned}
\left\|J_{1, n}\right\|_{k} & \leq \frac{\varepsilon}{3} \frac{n^{n+2}}{n !} \int_{1-\delta}^{1+\delta} e^{-n u} u^{n-1}(u-1)^{2} d u \\
& \leq \frac{\varepsilon}{3} \frac{n^{n+2}}{n !} \int_{0}^{+\infty} e^{-n u}\left(u^{n+1}-2 u^{n}+u^{n-1}\right) d u \\
& =\frac{\varepsilon}{3} \frac{n^{n+2}}{n !}\left(\frac{(n+1) !}{n^{n+2}}-2 \frac{n !}{n^{n+1}}+\frac{(n-1) !}{n^{n}}\right) \\
& =\frac{\varepsilon}{3}(n+1-2 n+n)=\frac{\varepsilon}{3}
\end{aligned}
$$

for all $n \in \mathbb{N}$ and $n>t w$.

Let

$$
J_{2, n}=\frac{n^{n+2}}{n ! t} \int_{0}^{1-\delta} e^{-n u} u^{n-1}(u-1) G(u t) d u
$$

and $\rho_{1}(u)=e^{-n u} u^{n-1}$, then $\rho_{1}^{\prime}(u)=e^{-n u} u^{n-2}((n-1)-n u)$, therefore if $n \delta>1$, i. e. $n>\frac{1}{\delta}, \rho_{1}^{\prime}(u)>0$ in $(0,1-\delta)$ and

$$
\begin{aligned}
\left\|J_{2, n}\right\|_{k} & \leq \frac{n^{n+2}}{n ! t} \int_{0}^{1-\delta} e^{-n u} u^{n-1}(u-1)\|G(t u)\|_{k} d u \\
& \leq \frac{n^{n+2}}{n ! t} e^{-n(1-\delta)}(1-\delta)^{n-1} \int_{0}^{1-\delta}(u-1)\|G(t u)\|_{k} d u .
\end{aligned}
$$

Let's take $a_{k}=\int_{0}^{1-\delta}(u-1)\|G(t u)\|_{k} d u$ and $b_{n}=\frac{n^{n+2}}{n ! t} e^{-n(1-\delta)}(1-\delta)^{n-1}$, then

$$
\begin{aligned}
\frac{b_{n+1}}{b_{n}} & =\frac{(n+1)^{n+3}}{n !(n+1)} \frac{e^{-(n+1)(1-\delta)}(1-\delta)^{n} n !}{n^{n+2} e^{-n(1-\delta)}(1-\delta)^{n-1}} \\
& =\frac{n}{n+1}\left(1+\frac{1}{n}\right)^{n+3} \frac{1-\delta}{e^{(1-\delta)}}
\end{aligned}
$$

and, since $\frac{b_{n+1}}{b_{n}} \rightarrow(1-\delta) e^{-\delta}<1$, then $\sum_{n>n_{0}} b_{n}$, where $n_{0}>\frac{1}{\delta}$, converges; therefore $b_{n} \rightarrow 0$ for $n \rightarrow+\infty$ and we get

$$
\left\|J_{2, n}\right\|_{k}<\frac{\varepsilon}{3}
$$

Let's define

$$
J_{3, n}=\frac{n^{n+2}}{n ! t} \int_{1+\delta}^{+\infty} e^{-n u} u^{n-1}(u-1) G(t u) d u
$$

if $\rho_{2}(u)=e^{-m u} u^{m}$, then $\rho_{2}^{\prime}(u)=-m e^{-m u} u^{m-1}(u-1)$; since $u>1+\delta$ we have $\rho_{2}^{\prime}(u)<0$ in $(1+\delta$, $+\infty)$, therefore $\rho_{2}(u)$ is decreasing on $(1+\delta,+\infty)$ for all $m \in \mathbb{N}, m>1$. Let's take $n_{0}>w t, n>n_{0}$, then

$$
\begin{aligned}
\left\|J_{3, n}\right\|_{k} & =\left\|\frac{n^{n+2}}{n ! t} \int_{1+\delta}^{+\infty} e^{-\left(n-n_{0}\right) u} u^{n-n_{0}} e^{-n_{0} u} u^{n_{0}-1}(u-1) G(t u) d u\right\|_{k} \\
& \leq \frac{n^{n+2}}{n ! t} e^{-\left(n-n_{0}\right)(1+\delta)}(1+\delta)^{n-n_{0}} \int_{1+\delta}^{+\infty} e^{-n_{0} u} u^{n_{0}-1}(u-1)\|G(t u)\|_{k} d u \\
& \leq \frac{n^{n+2}}{n ! t} e^{-\left(n-n_{0}\right)(1+\delta)}(1+\delta)^{n-n_{0}} \int_{1+\delta}^{+\infty} e^{-n_{0} u} u^{n_{0}-1}(u-1) M_{k} e^{w t u} d u
\end{aligned}
$$

Let's define $d_{n}=\frac{n^{n+2}}{n ! t} e^{-\left(n-n_{0}\right)(1+\delta)}(1+\delta)^{n-n_{0}}$ and $c_{n_{0}, k}=\int_{1+\delta}^{+\infty} e^{-n_{0} u} u^{n_{0}-1}(u-1) M_{k} e^{w t u} d u$, we get

$$
\left\|J_{3, n}\right\|_{k} \leq c_{n_{0}, k} d_{n}
$$

Since

$$
\frac{d_{n+1}}{d_{n}}=\left(1+\frac{1}{n}\right)^{n+2} e^{-1-\delta}(1+\delta)
$$


then $\frac{d_{n+1}}{d_{n}} \rightarrow e^{-\delta}(1+\delta)<1$, moreover $\sum_{n>n_{0}} d_{n}$ converges and we obtain $d_{n} \rightarrow 0$ for $n \rightarrow+\infty$ and

$$
\left\|J_{3, n}\right\|_{k} \leq \frac{\varepsilon}{3}
$$

Since

$$
J_{n}=J_{1, n}+J_{2, n}+J_{3, n}
$$

we get

$$
\left\|J_{n}\right\|_{k} \rightarrow 0
$$

for $n \rightarrow+\infty$, for all $k \in \mathbb{N}$; then

$$
\mathfrak{F}-\lim _{n \rightarrow+\infty} J_{n}=0
$$

and

$$
\mathfrak{F}-\lim _{n \rightarrow+\infty}(-1)^{n} \frac{1}{n !}\left(\frac{n}{t}\right)^{n+1} \widehat{f}^{(n)}\left(\frac{n}{t}\right)=f(t) .
$$

5. The $\mathfrak{F} s \operatorname{Lip}_{0}([0,+\infty), \mathfrak{F})$ Space and the $\mathcal{L}_{B}(X, \mathfrak{F})$ Space

Let $\mathfrak{F}$ a Fréchet space and $f:[0,+\infty) \rightarrow \mathfrak{F}$, we define

$$
[f]_{0,1, \widetilde{Y}}=\sup _{t>s \geq 0}\left\{\frac{|f(t)-f(s)|_{\mathfrak{Y}}}{|t-s|}\right\}
$$

then

$$
\operatorname{Lip}_{0}([0,+\infty), \mathfrak{F})=\left\{f:[0,+\infty) \rightarrow \mathfrak{F}: f(0)=0,[f]_{0,1, \mathfrak{F}}<+\infty\right\}
$$

Proposition 8. $[\cdot]_{0,1, \widetilde{\mho}}$ is a quasi-norm.

Proposition 9. $\left(\right.$ Lip $\left._{0}([0,+\infty), \mathfrak{F}),[\cdot]_{0,1, \widetilde{\mho}}\right)$ is a quasi-normed metric space.

We define

$$
[f]_{0,1, k}=\sup _{t>s \geq 0}\left\{\frac{\|f(t)-f(s)\|_{k}}{|t-s|}\right\}
$$

and

$$
\mathfrak{F} \operatorname{sLip}_{0}([0,+\infty), \mathfrak{F})=\left\{f:[0,+\infty) \rightarrow \mathfrak{F}: f(0)=0,[f]_{0,1, k}<+\infty \quad \forall k \in \mathbb{N}\right\} .
$$

Proposition 10. $[\cdot]_{0,1, k}$ are semi-norms.

Proposition 11. The function define by

$$
|f|_{\widetilde{\mho} s i p_{0}([0,+\infty), \mathfrak{\mho})}=\sum_{k=0}^{+\infty} \frac{[f]_{0,1, k}}{2^{k}\left(1+[f]_{0,1, k}\right)} \quad \forall f \in \mathfrak{F} s \operatorname{Lip}_{0}([0,+\infty), \mathfrak{F})
$$

is a qusi-norm.

Proposition 12. $\left(\widetilde{F} s L i p_{0}([0,+\infty), \mathfrak{F}),|\cdot|_{\widetilde{s} s i p_{0}([0,+\infty), \mathscr{\mho})}\right)$ is a quasi-normed metric space.

Let $\mathfrak{F}$ a Fréchet space, $X$ a Banach space and $f: X \rightarrow \mathfrak{F}$ a linear continuous operator; then for all $k \in \mathbb{N}$ there exists $C_{k}>0$ such that

$$
\|f(x)\|_{\widetilde{\gamma}, k} \leq C_{k}\|x\|_{X} \quad \forall x \in X .
$$

Definition 28. Let $\mathfrak{F}$ a Fréchet space, $X$ a Banach space and $f \in \mathcal{L}(X, \mathfrak{F})$ we define

$$
\|f\|_{\mathcal{L}(X, \mathfrak{\mho}), k}=\sup _{\substack{x \in X \\\|x\|_{X} \leq 1}}\left\{\|f(x)\|_{\widetilde{r}, k}\right\}
$$

and

$$
|f|_{\mathcal{L}(X, \mathscr{Y})}=\sum_{k=0}^{+\infty} \frac{\|f\|_{\mathcal{L}(X, \mathfrak{F}), k}}{2^{k}\left(1+\|f\|_{\mathcal{L}(X, \mathscr{\mho}), k}\right)}
$$


Proposition 13. $\|\cdot\|_{\mathcal{L}(X, \mathscr{\mho}), k}$ are semi-norms and $|\cdot|_{\mathcal{L}(X, \mathscr{Y})}$ is a quasi-norm.

Proposition 14. $\left(\mathcal{L}(X, \mathfrak{F}),|\cdot|_{\mathcal{L}(X, \mathscr{F})}\right)$ is a quasi-normed complete metric space.

Proof. Let $\left\{T_{n}\right\}_{n \in \mathbb{N}} \subset \mathcal{L}(X, \mathfrak{F})$ be a Cauchy sequence.

Fix $\eta>0$ and $\varepsilon<\frac{\eta}{2^{k}(1+\eta)}$ then there exists $n_{\varepsilon} \in \mathbb{N}$ such that for all $n, m>n_{\varepsilon}$

$$
\left|T_{n}-T_{m}\right|_{\mathcal{L}(X, \mathscr{\mho})}<\varepsilon
$$

and

$$
\left\|T_{n}-T_{m}\right\|_{\mathcal{L}(X, \mathfrak{\mho}), k}<\eta
$$

$\left\{\left\|T_{n}\right\|_{\mathcal{L}(X, \widetilde{\mho}), k}\right\}_{n \in \mathbb{N}} \subset \mathbb{R}$ is a Cauchy sequence, then is bounded; moreover

$$
\left\|T_{n} x-T_{m} x\right\|_{k} \leq\left\|T_{n}-T_{m}\right\|_{\mathcal{L}(X, \widetilde{\mho}), k}\|x\|_{X}
$$

then $\left\{T_{n} x\right\}_{n \in \mathbb{N}} \subset \mathfrak{F}$ is a Cauchy sequence in $\mathfrak{\mho}$ and there exists $\widetilde{y} \in \mathfrak{F}$ such that $T_{n} x \rightarrow \widetilde{y}$ for $n \rightarrow+\infty$. Let's define

$$
T: X \rightarrow \mathfrak{F}
$$

by

$$
T x=\tilde{y} .
$$

Since

$$
\left\|T_{n} x\right\|_{k} \leq\left\|T_{n}\right\|_{\mathcal{L}(X, \widetilde{\mho}), k}\|x\|_{X} \leq C_{k}\|x\|_{X}
$$

by theorem (2.1) and corollary (2.1) of [19], we have $T \in \mathcal{L}(X, \mathfrak{F})$.

Let's consider

$$
\left\|T_{n} x-T_{m} x\right\|_{k} \leq \eta\|x\|_{X} \quad \forall n, m>n_{\eta}
$$

then, since $T_{m} x \rightarrow T x$,

$$
\left\|T_{n} x-T x\right\|_{k} \leq \eta\|x\|_{X} \quad \forall n>n_{\eta}
$$

and we have

$$
\left\|T_{n}-T\right\|_{\mathcal{L}(X, \mathscr{\mho}), k} \leq \eta
$$

then $T_{n} \rightarrow T$ in $\left(\mathcal{L}(X, \mathfrak{F}),|\cdot|_{\mathcal{L}(X, \mathfrak{F})}\right)$ for $n \rightarrow+\infty$.

Definition 29. Let $\mathfrak{F}$ a Fréchet space, $X$ a Banach space and $f: X \rightarrow \mathfrak{F}$ a linear operator; $f$ is bounded if exists a real costant $K$ such that

$$
|f(x)|_{\widetilde{\gamma}} \leq K\|x\|_{X} \quad \forall x \in X .
$$

Lemma 4. Let $\mathfrak{F}$ a Fréchet space, $X$ a Banach space and $f: X \rightarrow \mathfrak{F}$ a linear operator; if it is bounded then it is continuous.

Proposition 15. Let $\mathfrak{F}$ a Fréchet space and X a Banach space; then

1. $\mathcal{L}_{B}(X, \mathfrak{F})=\{f: X \rightarrow \mathfrak{F}: f$ is linear and bounded $\} \subset \mathcal{L}(X, \mathfrak{F})$;

2. $|f|_{\mathcal{L}_{B}(X, \widetilde{\mho})}=\sup _{\substack{x \in X \\\|x\|_{X} \leq 1}}\left\{|f(x)|_{\mathfrak{F}}\right\}$ is a quasi-norm;

3. $\left(\mathcal{L}_{B}(X, \mathfrak{F}),|\cdot|_{\mathcal{L}_{B}(X, \widetilde{\mho})}\right)$ is a quasi-normed, complete metric space.

Proof. Let $\left\{T_{n}\right\}_{n \in \mathbb{N}} \subset \mathcal{L}_{B}(X, \mathfrak{F})$ be a Cauchy sequence.

Let's fix $\varepsilon>0$ and $x \in X$; then

$$
\left|T_{n} x-T_{m} x\right|_{\mathfrak{F}} \leq\left|T_{n}-T_{m}\right|_{\mathcal{L}_{B}(X, \mathfrak{F})}\|x\|_{X} \leq \varepsilon\|x\|_{X}
$$

for all $n, m>n_{\varepsilon} \in \mathbb{N}$. 
By (5.19) we have that $\left\{T_{n} x\right\}_{n \in \mathbb{N}} \subset \mathfrak{F}$ is a Cauchy sequence and there exists $\widetilde{y} \in \mathfrak{F}$ such that $T_{n} x \rightarrow \widetilde{y}$ for $n \rightarrow+\infty$. Let's define

$$
T: X \rightarrow \mathfrak{r}
$$

by

$$
T x=\tilde{y} .
$$

We see that $T$ is linear and that $\left|T_{n}\right|_{\mathcal{L}_{B}(X, \widetilde{\mho})}<C$ for all $n \in \mathbb{N}$ because $\left\{\left|T_{n}\right|_{\mathcal{L}_{B}(X, \widetilde{\mho})}\right\}_{n \in \mathbb{N}} \subset \mathbb{R}$ is a Cauchy sequence; by theorem (2.1) and corollary (2.1) of [19] we obtain that $T \in \mathcal{L}(X, \mathfrak{F})$. Let's consider $|T x|_{\mathscr{F}}$ then

$$
\begin{aligned}
|T x|_{\mathfrak{F}} & \leq\left|T_{n} x-T x\right|_{\mathfrak{F}}+\left|T_{n} x\right|_{\mathfrak{F}} \\
& \leq \varepsilon+\left|T_{n} x\right|_{\mathfrak{F}} \\
& \leq \varepsilon+\left|T_{n}\right|_{\mathcal{L}_{B}(X, \mathfrak{F})}\|x\|_{X} \\
& \leq \varepsilon+C\|x\|_{X}
\end{aligned}
$$

and for $\varepsilon \downarrow 0$ we have $|T x|_{\tilde{\mho}} \leq C\|x\|_{X}$. By (5.22) we obtain

$$
\left|T_{n} x-T x\right|_{\mathfrak{\wp}} \leq \varepsilon\|x\|_{X}
$$

for all $n>n_{\varepsilon}$; then $T_{n} \rightarrow T$ in $\left(\mathcal{L}_{B}(X, \mathfrak{F}),|\cdot|_{\mathcal{L}_{B}(X, \widetilde{\mho})}\right)$.

\section{A Real Representation Theorem (I)}

Theorem 8. There exists a unique isomorphism $\Phi_{S}: F \mapsto T_{F}$ from Lip $\operatorname{Lip}_{0}([0,+\infty), \mathfrak{F})$ onto $\mathcal{L}_{B}\left(L^{1}([0,+\infty), \mathbb{C}), \mathfrak{F}\right)$ such that

$$
T_{F \chi[0, t]}=F(t)
$$

for all $t \geq 0$ and $F \in \operatorname{Lip}_{0}([0,+\infty), \mathfrak{F})$. Moreover,

$$
T_{F} g=\lim _{t \rightarrow+\infty} \int_{0}^{t} g(s) d F(s)=\int_{0}^{+\infty} g(s) d F(s)
$$

for all continuous functions $g \in L^{1}([0,+\infty), \mathbb{C})$.

Proof. (Step 1). Let $D=\operatorname{span}\left\{\chi_{[0, t]}: t>0\right\}$; it is dense in $L^{1}([0,+\infty), \mathbb{C})$, moreover $\forall f \in D$ exists a unique representation,

$$
f=\sum_{i=1}^{n} \alpha_{i} \chi_{\left[t_{i-1}, t_{i}\right)}
$$

where $0=t_{0} \leq t_{1} \leq \ldots \leq t_{n}=t$ and $\alpha_{i} \in \mathbb{C}$.

Let $F \in \operatorname{Lip}(\mathbb{R}, \mathfrak{F}) \cap C^{\infty}(\mathbb{R}, \mathfrak{F})$; we define $T_{F}: D \rightarrow \mathfrak{F}$ by

$$
T_{F}(f)=T_{F}\left(\sum_{i=1}^{n} \alpha_{i} \chi_{\left[t_{i-1}, t_{i}\right)}\right)=\sum_{i=1}^{n} \alpha_{i}\left(F\left(t_{i}\right)-F\left(t_{i-1}\right)\right) ;
$$


then

$$
\begin{aligned}
\left|T_{F}(f)\right|_{\widetilde{F}} & =\left|\sum_{i=1}^{n} \alpha_{i}\left(F\left(t_{i}\right)-F\left(t_{i-1}\right)\right)\right|_{\widetilde{F}} \\
& \leq \sum_{i=1}^{n}\left|\alpha_{i}\left(F\left(t_{i}\right)-F\left(t_{i-1}\right)\right)\right|_{\mathfrak{F}} \\
& =\sum_{i=1}^{n}\left|\alpha_{i} \int_{t_{i-1}}^{t_{i}} F^{\prime}(s) d s\right|_{\widetilde{F}} \\
& =\sum_{i=1}^{n}\left|\frac{\alpha_{i}}{\left|\alpha_{i}\right|} \int_{t_{i-1}}^{t_{i}}\right| \alpha_{i}\left|F^{\prime}(s) d s\right|_{\widetilde{F}} \\
& =\sum_{i=1}^{n}\left|\int_{t_{i-1}}^{t_{i}}\right| \alpha_{i}\left|F^{\prime}(s) d s\right|_{\widetilde{F}} \\
& =\sum_{i=1}^{n}\left|\int_{\left|\alpha_{i}\right| t_{i-1}}^{\left|\alpha_{i}\right| t_{i}} F^{\prime}\left(\frac{s}{\left|\alpha_{i}\right|}\right) d s\right|_{\widetilde{F}} \\
& \leq\left.\sum_{i=1}^{n}\left|\int_{\left|\alpha_{i}\right| t_{i} \mid t_{i-1}}\right| F^{\prime}\left(\frac{s}{\left|\alpha_{i}\right|}\right)\right|_{\widetilde{F}} d s \\
& \leq \sum_{i=1}^{n}\left|\alpha_{i}\right|\left(t_{i}-t_{i-1}\right)\|F\|_{L i p(\mathbb{R}, \widetilde{F})} \\
& =\|f\|_{L^{1}([0,+\infty), \mathbb{C})}\|F\|_{L i p(\mathbb{R}, \widetilde{F})}
\end{aligned}
$$

Let $F \in \operatorname{Lip}_{0}([0,+\infty), \mathfrak{F})$ then we define

$$
\bar{F}(t)= \begin{cases}F(t) & \text { if } t \geq 0 \\ 0 & \text { if } t<0\end{cases}
$$

and we have $\bar{F} \in \operatorname{Lip}(\mathbb{R}, \mathfrak{F})$ and

$$
\|\bar{F}\|_{L i p(\mathbb{R}, \widetilde{F})} \leq\|F\|_{L i p_{0}([0,+\infty), \widetilde{F})}
$$

Let $\varrho_{n} * \bar{F} \in C^{\infty}(\mathbb{R}, \mathfrak{F})$ then

$$
\begin{aligned}
\left|\left(\varrho_{n} * \bar{F}\right)(x)-\left(\varrho_{n} * \bar{F}\right)(y)\right|_{F} & =\left|\int_{\mathbb{R}} \varrho_{n}(x-s) \bar{F}(s) d s-\int_{\mathbb{R}} \varrho_{n}(y-s) \bar{F}(s) d s\right|_{F} \\
& =\left|\int_{B_{\frac{1}{n}}(0)} \varrho_{n}(s)[\bar{F}(x-s)-\bar{F}(y-s)] d s\right|_{F} \\
& \leq n \int_{B_{\frac{1}{n}}(0)}|[\bar{F}(x-s)-\bar{F}(y-s)]|_{F} d s \\
& =|x-y| n \int_{B_{\left.\frac{1}{(}\right)}} \frac{\mid\left[\bar{F}(x-s)-\bar{F}(y-s) \|_{F}\right.}{|x-y|} d s \\
& \leq 2|x-y|\|\bar{F}\|_{L i p(\mathbb{R}, \widetilde{Y})} \\
& \leq 2|x-y|\|F\|_{L i p_{0}([0,+\infty), \mathscr{F}) .}
\end{aligned}
$$

By (6.7) and (6.8) we have

$$
\varrho_{n} * \bar{F} \in \operatorname{Lip}(\mathbb{R}, \mathfrak{F}) \cap C^{\infty}(\mathbb{R}, \mathfrak{F})
$$

and

$$
\begin{aligned}
\left|T_{\varrho_{n} * \bar{F}}(f)\right|_{\widetilde{F}} & \leq\|f\|_{L^{1}([0,+\infty), \mathbb{C})}\left\|\varrho_{n} * \bar{F}\right\|_{L i p(\mathbb{R}, \mathscr{F})} \\
& \leq 2\|f\|_{L^{1}([0,+\infty), \mathbb{C})}\|F\|_{L i p_{0}([0,+\infty), \mathscr{F})} .
\end{aligned}
$$

We proof that for every $x \in \mathbb{R}$

$$
\left|\varrho_{n} * \bar{F}(x)-\bar{F}(x)\right|_{\mathfrak{F}} \rightarrow 0 \text { for } n \rightarrow+\infty \text {. }
$$


Let $x \in \mathbb{R}$

$$
\begin{aligned}
\left|\varrho_{n} * \bar{F}(x)-\bar{F}(x)\right|_{\mathfrak{F}} & =\left|\int_{\mathbb{R}} \varrho_{n}(x-s)[\bar{F}(s)-\bar{F}(x)] d s\right|_{\widetilde{F}} \\
& \leq \int_{B_{\frac{1}{n}}(0)}\left|\varrho_{n}(s)[\bar{F}(x-s)-\bar{F}(x)]\right|_{\mathfrak{F}} d s \\
& =\int_{B_{\frac{1}{n}}(0)}\left|n \frac{\varrho(n s)}{\iint_{\mathbb{R}} \varrho(z) d z}[\bar{F}(x-s)-\bar{F}(x)]\right|_{\widetilde{F}} d s \\
& \leq \int_{B_{1}(0)}\left|\frac{\varrho(s)}{\int_{\mathbb{R}} \varrho(z) d z}\left[\bar{F}\left(x-\frac{s}{n}\right)-\bar{F}(x)\right]\right|_{\widetilde{F}} d s
\end{aligned}
$$

then by the scalar dominated convergenge theorem we have (6.11), moreover

$$
\begin{aligned}
\left|T_{\varrho_{n} * \bar{F}}(f)-T_{F}(f)\right|_{\mathfrak{r}} & =\left|\sum_{i=1}^{n} \alpha_{i}\left(\varrho_{n} * \bar{F}\left(t_{i}\right)-\varrho_{n} * \bar{F}\left(t_{i-1}\right)\right)-\sum_{i=1}^{n} \alpha_{i}\left(F\left(t_{i}\right)-F\left(t_{i-1}\right)\right)\right|_{\overparen{F}} \\
& =\left|\sum_{i=1}^{n} \alpha_{i}\left(\varrho_{n} * \bar{F}\left(t_{i}\right)-F\left(t_{i}\right)-\varrho_{n} * \bar{F}\left(t_{i-1}\right)+F\left(t_{i-1}\right)\right)\right|_{\widetilde{F}} \\
& \leq \sum_{i=1}^{n} \max \left\{1,\left|\alpha_{i}\right|\right\}\left[\left|\varrho_{n} * \bar{F}\left(t_{i}\right)-F\left(t_{i}\right)\right|_{\mathfrak{\mho}}+\left|\varrho_{n} * \bar{F}\left(t_{i-1}\right)-F\left(t_{i-1}\right)\right|_{\overparen{F}}\right]
\end{aligned}
$$

then $\left|T_{\varrho_{n} * \bar{F}}(f)-T_{F}(f)\right|_{\mathfrak{F}} \rightarrow 0$ for $n \rightarrow+\infty$ and

$$
\lim _{n \rightarrow+\infty}\left|T_{\varrho_{n} * \bar{F}}(f)\right|_{\mathfrak{F}}=\left|T_{F}(f)\right|_{\mathfrak{F}} .
$$

By (6.11) and (6.12), if $f \in D$, we have

$$
\left|T_{F}(f)\right|_{\mathfrak{F}}=\lim _{n \rightarrow+\infty}\left|T_{\varrho_{n} * \bar{F}}(f)\right|_{\mathfrak{F}} \leq 2\|f\|_{L^{1}([0,+\infty), \mathbb{C})}\|F\|_{L i p_{0}([0,+\infty), \mathfrak{Y})} .
$$

(Step 2). Conversely, if $T \in \mathcal{L}_{B}\left(L^{1}([0,+\infty), \mathbb{C}), \mathfrak{F}\right)$ we define

$$
F(t)=T \chi_{[0, t)} \quad \forall t \geq 0 .
$$

We proof that $F \in \operatorname{Lip}_{0}([0,+\infty), \mathfrak{F})$; by (6.14) we obtain $F(0)=T(0)=0$; let $t>s \geq 0$, then

$$
\begin{aligned}
|F(t)-F(s)|_{\mathfrak{F}} & \leq\left|T \chi_{[0, t)}-T \chi_{[0, s)}\right|_{\mathfrak{F}} \\
& =\left|T\left(\chi_{[0, t)}-\chi_{[0, s)}\right)\right|_{\mathfrak{F}} \\
& =\left|T\left(\chi_{[s, t)}\right)\right|_{\mathfrak{F}} \\
& \leq\|T\|_{\mathcal{L}_{B}\left(L^{1}([0,+\infty), \mathbb{C}), \mathfrak{\mho}\right)}\left\|\chi_{[s, t)}\right\|_{L^{1}([0,+\infty), \mathbb{C})} \\
& =\|T\|_{\mathcal{L}_{B}\left(L^{1}([0,+\infty), \mathbb{C}), \mathfrak{F}\right)}(t-s),
\end{aligned}
$$

$F \in \operatorname{Lip}_{0}([0,+\infty), \mathfrak{F})$ and

$$
\|F\|_{L i p_{0}([0,+\infty), \widetilde{\mho})} \leq\|T\|_{\mathcal{L}_{B}\left(L^{1}([0,+\infty), \mathbb{C}), \mathscr{\mho}\right)} \cdot
$$

We proof that $T_{F}=T$; let $f \in D$ then

$$
\begin{aligned}
T_{F}(f) & =T_{F}\left(\sum_{i=1}^{n} \alpha_{i} \chi_{\left[t_{i-1}, t_{i}\right)}\right) \\
& =\sum_{i=1}^{n} \alpha_{i}\left(F\left(t_{i}\right)-F\left(t_{i-1}\right)\right) \\
& =\sum_{i=1}^{n} \alpha_{i}\left(T \chi_{\left[0, t_{i}\right)}-T \chi_{\left[0, t_{i-1}\right)}\right) \\
& =\sum_{i=1}^{n} \alpha_{i}\left(T \chi_{\left[t_{i}, t_{i-1}\right)}\right) \\
& T\left(\sum_{i=1}^{n} \alpha_{i} \chi_{\left[t_{i}, t_{i-1}\right)}\right) \\
& =T(f)
\end{aligned}
$$


then $T=T_{F}$ on $D$; but $D$ is dende in $L^{1}([0,+\infty) ; \mathbb{C})$ then $T=T_{F}$. If $T=T_{G}$ we have $G=F$.

Let $g \in L^{1}([0,+\infty) ; \mathbb{C}) \cap C([0,+\infty) ; \mathbb{C}), F \in \operatorname{Lip} p_{0}([0,+\infty), \mathfrak{F})$ and $t>0$; if $\Pi$ denote a finite partition $0=t_{0}<t_{1}<$ $\ldots<t_{n}=t$ of $[0, t]$ with partitioning points $t_{i}$ and with some intermediate points $s_{i} \in\left[t_{i-1}, t_{i}\right]$, for $i=1, \ldots, n$, we denote by $|\Pi|=\max _{i=1, . ., n}\left\{\left(t_{i}-t_{i-1}\right)\right\}$ the norm of $\Pi$, then we have

$$
f_{\Pi}=\sum_{i=1}^{n} g\left(s_{i}\right) \chi_{\left[t_{i-1}, t_{i}\right)} \in D
$$

and

$$
\begin{aligned}
T_{F}\left(f_{\Pi}\right) & =T_{F}\left(\sum_{i=1}^{n} g\left(s_{i}\right) \chi_{\left[t_{i-1}, t_{i}\right)}\right) \\
& =\sum_{i=1}^{n} g\left(s_{i}\right)\left(F\left(t_{i}\right)-F\left(t_{i-1}\right)\right) \\
& =S(g, F, \Pi) .
\end{aligned}
$$

We proof that $f_{\Pi} \rightarrow g \chi_{[0, t)}$ in $L^{1}([0,+\infty) ; \mathbb{C})$ for $|\Pi| \rightarrow 0$

$$
\begin{aligned}
\left\|\sum_{i=1}^{n} g\left(s_{i}\right) \chi_{\left[t_{i-1}, t_{i}\right)}-g \chi_{[0, t)}\right\|_{L^{1}([0,+\infty) ; \mathbb{C})} & =\int_{0}^{t}\left|\sum_{i=1}^{n} g\left(s_{i}\right) \chi_{\left[t_{i-1}, t_{i}\right)}(s)-g(s) \chi_{[0, t)}(s)\right| d s \\
& =\int_{0}^{t}\left|\sum_{i=1}^{n}\left(g\left(s_{i}\right)-g(s)\right) \chi_{\left[t_{i-1}, t_{i}\right)}(s)\right| d s \\
& \leq \int_{0}^{t} \sum_{i=1}^{n}\left|g\left(s_{i}\right)-g(s)\right| \chi_{\left[t_{i-1}, t_{i}\right)}(s) d s
\end{aligned}
$$

then by the Cantor's theorem we have

$$
\left\|\sum_{i=1}^{n} g\left(s_{i}\right) \chi_{\left[t_{i-1}, t_{i}\right)}-g \chi_{[0, t)}\right\|_{L^{1}([0,+\infty) ; \mathbb{C})} \rightarrow 0
$$

if $|\Pi| \rightarrow 0$. By (6.19) and (6.21) we obtain

$$
T_{F}\left(g \chi_{[0, t)}\right)=\int_{0}^{t} g(s) d F(s)
$$

then for $t \rightarrow+\infty$, since $g \chi_{[0, t)} \rightarrow g$ in $L^{1}([0,+\infty) ; \mathbb{C})$,

$$
T_{F}(g)=\int_{0}^{+\infty} g(s) d F(s) .
$$

Proposition 16. $\left(\operatorname{Lip}_{0}([0,+\infty), \mathfrak{F}),|\cdot|_{L i p_{0}([0,+\infty), \widetilde{\mho})}\right)$ is a quasi-normed, complete metric space.

Proof. By theorem (8) and proposition (15).

\section{A Real Representation Theorem (II)}

Theorem 9. There exists a unique isometric isomorphism $\Phi_{S}: F \mapsto T_{F}$ from $\mathfrak{F} \operatorname{sip} p_{0}([0,+\infty)$, $)$ onto $\mathcal{L}\left(L^{1}([0,+\infty), \mathbb{C}), \mathfrak{\mho}\right)$ such that

$$
T_{F} \chi_{[0, t]}=F(t)
$$

for all $t \geq 0$ and $F \in \mathfrak{F} s \operatorname{Lip}_{0}([0,+\infty), \mathfrak{F})$. Moreover,

$$
T_{F} g=\lim _{t \rightarrow+\infty} \int_{0}^{t} g(s) d F(s)=\int_{0}^{+\infty} g(s) d F(s)
$$

for all continuous functions $g \in L^{1}([0,+\infty), \mathbb{C})$. 
Proof. (Step 1). Let $D=\operatorname{span}\left\{\chi_{[0, t]}: t>0\right\}$; it is dense in $L^{1}([0,+\infty), \mathbb{C})$, moreover $\forall f \in D$ exists a unique representation,

$$
f=\sum_{i=1}^{n} \alpha_{i} \chi_{\left[t_{i-1}, t_{i}\right)}
$$

where $0=t_{0} \leq t_{1} \leq \ldots \leq t_{n}=t$ and $\alpha_{i} \in \mathbb{C}$.

Let $F \in \mathfrak{F} \operatorname{sip}_{0}([0 .+\infty), \mathfrak{F})$; we define $T_{F}: D \rightarrow \mathfrak{F}$ by

$$
T_{F}(f)=T_{F}\left(\sum_{i=1}^{n} \alpha_{i} \chi_{\left[t_{i-1}, t_{i}\right)}\right)=\sum_{i=1}^{n} \alpha_{i}\left(F\left(t_{i}\right)-F\left(t_{i-1}\right)\right) ;
$$

then

$$
\begin{aligned}
\left\|T_{F}(f)\right\|_{k} & =\left\|\sum_{i=1}^{n} \alpha_{i}\left(F\left(t_{i}\right)-F\left(t_{i-1}\right)\right)\right\|_{k} \\
& \leq \sum_{i=1}^{n} \alpha_{i}\left\|F\left(t_{i}\right)-F\left(t_{i-1}\right)\right\|_{k} \\
& \leq \operatorname{Lip} p_{0, k}(F) \sum_{i=1}^{n} \alpha_{i}\left(t_{i}-t_{i-1}\right) \\
& =\operatorname{Lip}_{0, k}(F)\|f\|_{L^{1}([0,+\infty), \mathbb{C})} .
\end{aligned}
$$

Let $f \in L^{1}([0,+\infty), \mathbb{C})$ and $\varepsilon>0$ there exists $g_{\varepsilon, k} \in D$ such that $\|f-g\|_{L^{1}([0,+\infty), \mathbb{C})}<\frac{\varepsilon}{\operatorname{Lip}_{k}(F)}$, by (4.5) for all $k \in \mathbb{N}$ we have $\left\|T_{F}(f-g)\right\|_{k} \leq \varepsilon, T_{F}$ has a unique extension and

$$
\left\|T_{F}(f)\right\|_{k} \leq \operatorname{Lip}_{0, k}(F)
$$

Let

$$
\Phi_{S}: \mathfrak{F} \operatorname{sip}_{0}([0,+\infty), \mathfrak{F}) \rightarrow \mathcal{L}\left(L^{1}([0,+\infty), \mathbb{C}), \mathfrak{F}\right)
$$

defined by $F \mapsto T_{F}$; then $\Phi_{S}$ is linear and $\left\|\Phi_{S}(F)\right\|_{k} \leq L i p_{0, k}(F)$ for all $k \in \mathbb{N}$.

(Step 2). Conversely, if $T \in \mathcal{L}\left(L^{1}([0,+\infty), \mathbb{C})\right.$, $)$ we define

$$
F(t)=T \chi_{[0, t)} \quad \forall t \geq 0 .
$$

We proof that $F \in \mathfrak{F} s \operatorname{Lip}_{0}([0,+\infty), \mathfrak{F})$. By (7.8) $F(0)=T(0)=0$ and if $t>s \geq 0$ we have

$$
\begin{aligned}
\|F(t)-F(s)\|_{k} & =\left\|T \chi_{[0, t)}-T \chi_{[0, s)}\right\|_{k} \\
& =\left\|T \chi_{[s, t}\right\|_{k} \\
& \leq\|T\|_{k}\left\|\chi_{[s, t)}\right\|_{L^{1}([0,+\infty), \mathbb{C})} \\
& \leq\|T\|_{k}(t-s)
\end{aligned}
$$

and

$$
\operatorname{Lip}_{0, k}(F) \leq\|T\|_{k}
$$

Let $f \in D$ then

$$
\begin{aligned}
T_{F}(f) & =T_{F}\left(\sum_{i=1}^{n} \alpha_{i} \chi_{\left[t_{i-1}, t_{i}\right)}\right) \\
& =\sum_{i=1}^{n} \alpha_{i}\left(F\left(t_{i}\right)-F\left(t_{i-1}\right)\right) \\
& =\sum_{i=1}^{n} \alpha_{i}\left(T \chi_{\left[0, t_{i}\right)}-T \chi_{\left[0, t_{i-1}\right)}\right) \\
& =\sum_{i=1}^{n} \alpha_{i}\left(T \chi_{\left[t_{i}, t_{i-1}\right)}\right) \\
& T\left(\sum_{i=1}^{n} \alpha_{i} \chi_{\left[t_{i}, t_{i-1}\right)}\right) \\
& =T(f),
\end{aligned}
$$

then $T=T_{F}$ on $D$; but $D$ is dense in $L^{1}([0,+\infty) ; \mathbb{C})$ then $T=T_{F}$ and if $T=T_{G}$ we have $G=F$. 
Let $g \in L^{1}([0,+\infty) ; \mathbb{C}) \cap C([0,+\infty) ; \mathbb{C}), F \in \mathfrak{F} s \operatorname{sip}_{0}([0,+\infty), \mathfrak{F})$ and $t>0$; if $\Pi$ denote a finite partition $0=t_{0}<t_{1}<$ $\ldots<t_{n}=t$ of $[0, t]$ with partitioning points $t_{i}$ and with some intermediate points $s_{i} \in\left[t_{i-1}, t_{i}\right]$, for $i=1, \ldots, n$, we denote by $|\Pi|=\max _{i=1, \ldots, n}\left\{\left(t_{i}-t_{i-1}\right)\right\}$ the norm of $\Pi$, then we have

$$
f_{\Pi}=\sum_{i=1}^{n} g\left(s_{i}\right) \chi_{\left[t_{i-1}, t_{i}\right)} \in D
$$

and

$$
\begin{aligned}
T_{F}\left(f_{\Pi}\right) & =T_{F}\left(\sum_{i=1}^{n} g\left(s_{i}\right) \chi_{\left[t_{i-1}, t_{i}\right)}\right) \\
& =\sum_{i=1}^{n} g\left(s_{i}\right)\left(F\left(t_{i}\right)-F\left(t_{i-1}\right)\right) \\
& =S(g, F, \Pi) .
\end{aligned}
$$

We proof that $f_{\Pi} \rightarrow g \chi_{[0, t)}$ in $L^{1}([0,+\infty) ; \mathbb{C})$ for $|\Pi| \rightarrow 0$

$$
\begin{aligned}
\left\|\sum_{i=1}^{n} g\left(s_{i}\right) \chi_{\left[t_{i-1}, t_{i}\right)}-g \chi_{[0, t)}\right\|_{L^{1}([0,+\infty) ; \mathbb{C})} & =\int_{0}^{t}\left|\sum_{i=1}^{n} g\left(s_{i}\right) \chi_{\left[t_{i-1}, t_{i}\right)}(s)-g(s) \chi_{[0, t)}(s)\right| d s \\
& =\int_{0}^{t}\left|\sum_{i=1}^{n}\left(g\left(s_{i}\right)-g(s)\right) \chi_{\left[t_{i-1}, t_{i}\right)}(s)\right| d s \\
& \leq \int_{0}^{t} \sum_{i=1}^{n}\left|g\left(s_{i}\right)-g(s)\right| \chi_{\left[t_{i-1}, t_{i}\right)}(s) d s
\end{aligned}
$$

then by the Cantor's theorem we have

$$
\left\|\sum_{i=1}^{n} g\left(s_{i}\right) \chi_{\left[t_{i-1}, t_{i}\right)}-g \chi_{[0, t)}\right\|_{L^{1}([0,+\infty) ; \mathbb{C})} \rightarrow 0
$$

if $|\Pi| \rightarrow 0$. By (7.15)

$$
T_{F}\left(g \chi_{[0, t)}\right)=\int_{0}^{t} g(s) d F(s)
$$

then for $t \rightarrow+\infty$, since $g \chi_{[0, t)} \rightarrow g$ in $L^{1}([0,+\infty) ; \mathbb{C})$,

$$
T_{F}(g)=\int_{0}^{+\infty} g(s) d F(s) .
$$

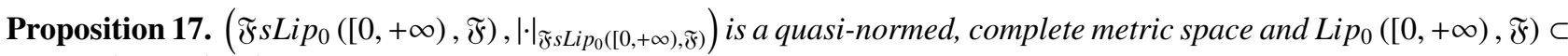
$\mathfrak{F} s \operatorname{Lip}_{0}([0,+\infty), \mathfrak{F})$.

Proof. By theorem (9) and proposition (14).

\section{References}

Arendt, W. (1987). Vector valued Laplace transforms and Cauchy problems, Israel J. Math., 59, 327-352. https://doi.org/10.1007/BF02774144

Arendt, W., Neubrander, F., \& Schlotterbeck, U. (1992). Interpolatio of semigroups and integrated semigroup, Semigroup Forum, 45, 26-37. https://doi.org/10.1007/BF03025746

Arendt, W., El-Mennaoui, O., \& Keyantuo, V. (1994). Local Integrated Semigroups: Evolution with Jumps of Regularity, J. Math. Anal. Appl., 186, 572-595. https://doi.org/10.1006/jmaa.1994.1318

Arendt, W., Batty, C. J. K., Hieber, M., \& Neubrander, F. (2001). Vector-valued Laplace Transforms and Cauchy Problems, Birkhäuser Verlag, Basel-Boston-Berlin. https://doi.org/10.1007/978-3-0348-5075-9 
Balabane, M., \& Emamirad, H. A. (1985). $L^{p}$ estimates for Schrödinger evolution equation, Trans. Amer. Math. Soc., 291, 357-373.

Carron, G., Coulhon, T., \& Ouhabaz, E. (2002). Gaussian estimates and -boundedeness of Risz mean, J. Evol. Equ., 2, 299-317. https://doi.org/10.1007/s00028-002-8090-1

Da Prato, G., \& Sinestrari, E. (1987). Differential operators with non dense domain, Ann. Scuola Norm. Sup. Pisa, XIV, 285-344.

Davies, E. B., \& Pang, M. M. (1987). The Cauchy problem and a generalization of the Hille-Yosida theorem, London Math. Soc., 55, 181-208. https://doi.org/10.1112/plms/s3-55.1.181

DeLaubenfels, R. (1993). Automatic well-posedness with the abstract Cauchy problem on a Fréchet space, J. London Math. Soc., 48(2), 526-536. https://doi.org/10.1112/j1ms/s2-48.3.526

Garding, L. (1953). Dirichlet's problem for linear elliptic partial differential equations, Math. Scand., 1. https://doi.org/10.7146/math.scand.a-10364

Granucci, T. (2005). Real representation theorems, integrated semigroups on Fréchet spaces and applications, Ulmer Seminare, 175-196.

Granucci, T. Integrated semigroups on Fréchet spaces II: $\mathfrak{F} s B S V([a, b], \mathfrak{F})$-space, $\mathfrak{F} s L i p_{0, w}([0,+\infty)$, $)$-space, isometric theorem and applications, forthcoming.

Hieber, M., \& Kellermann, H. (1989). Integrated semigroups, J. Funct. Anal. 84, 160-180. https://doi.org/10.1016/00221236(89)90116-X

Hieber, M. (1991). Laplace Transform and $\alpha$-Times Integrated Semigroups, Forum Math., 3, 595-612. https://doi.org/10.1515/form.1991.3.595

Hieber, M. (1991). Integrated semigroups and differential operators in $L^{p}$ spaces, Math. Ann., 291, 1-16. https://doi.org/10.1007/BF01445187

Hieber, M. (1991). Integrated semigroups and the Cauchy Problem for systems in $L^{p}$ spaces, J. Math. Anal. Appl., 162, 300-308. https://doi.org/10.1016/0022-247X(91)90196-7

Joseph, G. (1977). Boundedness and completeness in locally convex spaces and algebras, J. Austral. Math. Soc. Ser. A, 24, 50-63. https://doi.org/10.1017/S144678870002005X

Lax, P. D., \& Milgram, A. N. (1954). Parabolic equations, Contributions to the theory of partial differential equations, Princeton.

Miyadera, I. (1959). Semi-groups of operators in Fréchet space and applications to partial differential equations, Tohoku Math. J., 11(2), 162-183. https://doi.org/10.2748/tmj/1178244580

Miyadera, I. (1986). On the generator of exponentially C-semigroup, Proc. Japan Acad., 62, 239-242. https://doi.org/10.3792/pjaa.62.239

Neubrander, F. (1989). Integrated semigroups and their applications to the abstract Cauchy problem. Pacific J. Math. 135, 111-155. https://doi.org/10.2140/pjm.1988.135.111

Neubrander, F. (1989). Integrated semigroups and their applications to complete second order problem, Semigroup Forum, 38. 233-251. https://doi.org/10.1007/BF02573234

Okazawa, N., \& Tanaka, N. (1990). Local C-semigroups and local integrated semigroups, Proc. London Math. Soc., 61, 63-90.

Papaghiuc, N. (1080). Equations differentielles linéaires dans les espaces de Fréchet, Rev. Roumaine Math. Pures Appl., $25,83-88$.

Park, J. Y. (1990). Exponentially bounded $C$-semigroup in Fréchet space, Kobe J. Math., 7, 109-123.

Tanaka, N. (1987). On the exponentially bounded C-semigroup, Tokyo J. Math., 10, 107-117. https://doi.org/10.3836/tjm/1270141795

Tanaka, N. (1989). Holomorphic $C$-semigroups and holomorphic semigroups, Semigroup Forum, 38, 253-263.

Yosida, K. (1963). Holomorphic semi-groups in a locally convex linear topological space, Osaka Math. J., 15, 51-57. https://doi.org/10.1007/BF02573235

Yosida, K. (1965). Time dependent evolution equations in a locally convex space, Math. Ann., 162, 83-86. http- 


\section{s://doi.org/10.1007/BF01361935}

Yosida, K. (1965). Functional Analysis, Academic Press, New York.

Young, H. C. (1985). $C_{0}$-Semigroups on a Locally Convex Space, J. of Math. Anal. and Appl., 106, 293-320. https://doi.org/10.1016/0022-247X(85)90115-5

\section{Copyrights}

Copyright for this article is retained by the author(s), with first publication rights granted to the journal.

This is an open-access article distributed under the terms and conditions of the Creative Commons Attribution license (http://creativecommons.org/licenses/by/4.0/). 